\title{
MEAN CESÀRO SUMMABILITY OF LAGUERRE AND HERMITE SERIES $\left({ }^{1}\right)$
}

BY

\author{
EILEEN L. POIANI
}

ABSTRACT. The primary purpose of this paper is to prove inequalities of the type $\left\|\sigma_{n}(f, x) W(x)\right\|_{p} \leq C\|f(x) W(x)\|_{p}$ where $\sigma_{n}(f, x)$ is the $n$th $(C, 1)$ mean of the Laguerre or Hermite series of $f, W(x)$ is a suitable weight function of particular form, $C$ is a constant independent of $f(x)$ and $n$, and the norm is taken over $(0, \infty)$ in the Laguerre case and $(-\infty, \infty)$ in the Hermite case for $1 \leq p \leq \infty$. Both necessary and sufficient conditions for the se inequalities to remain valid are determined. For $p<\infty$ and $f(x) W(x) \in L^{p}$, mean summability re sults showing that $\lim _{n \rightarrow \infty}\left\|\left[\sigma_{n}(f, x)-f(x)\right] W(x)\right\|_{p}=0$ are derived by use of the appropriate density theorems. Detailed proofs are presented for the Laguerre expansions, and the analogous results for Hermite series follow as corollaries.

1. General introduction. This paper focuses on the proof of inequalities of the form $\left\|\sigma_{n}(f, x) W(x)\right\|_{p} \leq C\|f(x) W(x)\|_{p}$, where $\sigma_{n}$ is the $n$th $(C, 1)$ mean of the Laguerre or Hermite series of $f, W(x)$ is a weight function of particular form, and the $L^{p}$ norm is taken over $(0, \infty)$ in the Laguerre case and $(-\infty, \infty)$ in the Hermite case. Mean Cesàro summability results are derived by use of the appropriate density theorems. The study is undertaken first for Laguerre expansions, and the analogue for Hermite expansions is determined as a corollary.

As usual, $L_{n}^{a}(x)$, for $\alpha>-1$, denotes the Laguerre polynomial; $H_{n}(x)$, the Hermite polynomial. A brief historical account of recent developments in the investigation of such series follows.

Askey and Wainger [1] in 1965 verified that, for $4 / 3<p<4$ and $\alpha \geq 0$, Laguerre series are mean convergent in the $L^{p}$ spaces with weight function $\exp (-x / 2) x^{a / 2}$; Hermite series, with weight function $\exp \left(-x^{2} / 2\right)$. The verification that no functions exist to satisfy norm inequalities for Laguerre series for $1 \leq p \leq 4 / 3$ and $p \geq 4$ with the same weight function, $\exp (-x / 2) x^{\alpha / 2}$, is furnished in $[10]$.

In 1970, Muckenhoupt [11] not only generalized the results of [1] to all $a>-1$ in the Laguerre case, but also introduced new weight functions which

Presented to the Society, March 27, 1972; received by the editors August 30, 1971.

AMS (MOS) subject clas sifications (1970). Primary 42A56, 40G05; Secondary 33A65.

Key words and phrases. Mean Cesàro summability, Laguerre series, Hermite series, norm inequalities, weight function, Cesàro kernel estimates.

(1) The results in this paper constitute part of the author's Ph. D. dissertation, written under the direction of Professor B. Muckenhoupt at Rutgers University. 
yield mean convergence of both series for $1<p<\infty$. The results are also shown to be best possible in terms of the chosen weight functions. However, the conclusions of [11] were based on inequalities which required a larger weight function on the right side than the left. The conclusions reached in this work have the advantage of needing only one weight function.

General Cesàro means, $(C, \beta)$, for all real values $\beta$ except negative integers, are defined in [14, Vol. I, pp. 75-77]. The question of mean summability of Cesàro means of Laguerre and Hermite series for $\beta \geq 0$ was considered at length by Kogbetliantz [7]. His investigations were unfortunately based on the erroneous estimate, $L_{n}^{a}(x)=O\left(e^{x / 2} n^{a / 2-1 / 4} x^{-\alpha / 2-1 / 4}\right)$, as applied in the range $n \leq x<$ $e^{2} n[7, \$ 6]$. Kogbetliantz determined the Laguerre estimate by the "saddle-point method" [6, pp. 503-506] (also called "method of steepest descent").

Impetus to the study of these Cesàro means came from Campbell's representation of them by a differential operator in [21. In 1969, Ernst applied Campbell's technique to prove the $L^{p}$-norm convergence of the $(C, 1)$-means of Laguerre series for $1 \leq p<\infty$. Provided $\exp (-x r) f(x)$ is in $L^{i p}(0, \infty), 1 \leq p<\infty, 0<r<$ $1 / 2$, and $\alpha \geq 7 / 2$, Ernst shows in [5, Pp. 98-100] that there exists a positive constant $C$ such that

$$
\left\|\sigma_{n}(f, x) e^{-x / 2} x^{a / 2}\right\|_{p} \leq C\left\|f(x) e^{-x r}\right\|_{p}
$$

where \|\|$_{p}$ represents the usual $L^{p}$-norm on $(0, \infty)$ and $\sigma_{n}(f, x)$ is the $n$th Cesàro mean of a Laguerre series. The desired mean summability results follow from suitable density theorems.

As indicated, the present work is concerned primarily with proving inequalities of the type

$$
\left\|\sigma_{n}(f, x) W(x)\right\|_{p} \leq C\|f(x) W(x)\|_{p}
$$

where $W(x)$ is the weight function

$$
(x /(1+x))^{a}(1+x)^{b} e^{-x / 2} x^{a / 2}
$$

in the Laguerre case with $a$ and $b$ appropriate real numbers. The norm is taken over $(0, \infty)$ for $1 \leq p \leq \infty$ when $\sigma_{n}(f, x)$ represents the $n$th $(C, 1)$ mean of a Laguerre expansion. Only one weight function, $W(x)$, is needed in these inequalities. This fact substantially reduces Ernst's requirement that a larger weight function, $\exp (-x r)$ for $0<r<1 / 2$, be used on the right side than on the left side of his inequality.

Since $L_{n}^{a}(x)$ are associated with all $\alpha>-1$, the condition $\alpha \geq 7 / 2$ in the theorems of $[5]$ is unduly restrictive. The results here are valid for all $\alpha>-1$. Furthermore, because every Hermite polynomial is expressible in terms of a Laguerre one with parameters $\alpha= \pm 1 / 2$, the extended range in $\alpha$ permits 
immediate application to the mean Cesàro summability of Hermite series.

In $\$ 2$, necessary facts about Laguerre series and polynomials are stated.

Exact expressions and estimates for the Cesàro kernel of the Laguerre expansions appear in $\$ \$ 3$ and 4. The principal theorems regarding inequalities in (1.1) and mean Cesàro summability in the Laguerre case are stated and proved in $\$ 5$. Justification for the choice of the weight function, $W(x)$, is presented in $\$ \$ 6$ and 7. $\S \S 8$ and 9 give evidence that the conditions for summability are in a certain sense best possible. The results of earlier sections are applied to Hermite ex pansions in $\$ \$ 10$ and 11 .

2. Preliminaries about Laguerre series and polynomials. Given a function $f(x)$, its Laguerre series is defined by $\sum_{k=0}^{\infty} a_{k} L_{k}^{\alpha}(x), \alpha>-1$, where

$$
a_{k}=\frac{k !}{\Gamma(k+a+1)} \int_{0}^{\infty} e^{-x} x^{a} f(x) L_{k}^{a}(x) d x
$$

The partial sum of this series will be denoted by $s_{n}(f, x)$; the first Cesàro mean will be denoted by $\sigma_{n}(f, x)$, where, by definition [14, Vol. I, pp. 75-77],

$$
\sigma_{n}(f, x)=\frac{1}{n} \sum_{k=0}^{n-1}\left(\sum_{j=0}^{k} a_{j} L_{j}^{a}(x)\right) \text {. }
$$

The kernel of the partial sums will be defined by

$$
K_{n}(x, y)=\sum_{k=0}^{n} \frac{k !}{\Gamma(k+\alpha+1)} L_{k}^{a}(x) L_{k}^{\alpha}(y) .
$$

By the Christoffel-Darboux formula [12, (5.1.11), p. 101],

$$
K_{n}(x, y)=\frac{(n+1) !\left[L_{n}^{a}(x) L_{n+1}^{a}(y)-L_{n}^{a}(y) L_{n+1}^{a}(x)\right]}{\Gamma(n+\alpha+1)(x-y)} .
$$

Defining the kernel of the $(C, 1)$-means, $\sigma_{n}(f, x)$, to be

$$
K_{n, 1}=K_{n, 1}(x, y)=\frac{1}{n} \sum_{k=0}^{n-1} K_{k}(x, y)
$$

leads to the form

$$
\sigma_{n}(f, x)=\int_{0}^{\infty} K_{n, 1}(x, y) e^{-y} y^{a} f(y) d y .
$$

We remark that " $\sigma_{n}$ " is commonly used to denote the sum from 0 to $n$; however, the alteration used here is designed to simplify the explicit expression for $K_{n, 1}$ which will be given in (3.2).

In verifying inequalities of the form (1.1), the letter " $C$ " will be used to denote a generic constant whose value varies with the context. Mean Cesàro summability results will be derived from these inequalities whenever $f(x) W(x) \epsilon$ $L^{p}(0, \infty)$. 
There are myriad interesting identities associated with Laguerre polynomials $[3, \S 10.12]$. Those relevant to our results are included here.

$$
\begin{aligned}
L_{n}^{a}(x) & =L_{n+1}^{a}(x)-L_{n+1}^{a-1}(x), \\
L_{n}^{a-1}(x) & =\frac{a}{n+a} L_{n}^{a}(x)-\frac{x}{n+a} L_{n-1}^{a+1}(x), \\
L_{n}^{a+1}(x) & =L_{n}^{a}(x)+L_{n-1}^{a+1}(x), \\
L_{n-2}^{a+2}(x) & =\frac{-n}{x} L_{n}^{a}(x)+\left(\frac{\alpha+1}{x}-1\right) L_{n-1}^{a+1}(x) .
\end{aligned}
$$

The designation (5.-.-) refers to statements on Pp. 101-102 of [12]. In order of appearance, the identities are (5.1.13) with $\alpha=\alpha-1$ and $n=n+1$; the form $L_{n}^{a-1}(x)=L_{n}^{a}(x)-L_{n-1}^{a}(x)$ of (2.5) together with the second part of (5.1.14); (2.5) with $\alpha=\alpha+1$ and $n=n-1$; the first parts of (5.1.2) and (5.1.14).

All the Laguerre polynomial estimates which will be used are based on those of Erdélyi [4, pp. 240, 243] for $\alpha \geq 0$ with the range extended to $\alpha>-1$ by Muckenhoupt [9].

(2.9) Let $a>-1$ and $b<1$. There exists $n_{0}$ such that if $n \geq n_{0}$ and $0<x$ $\leq b \nu$, then $L_{n}^{a}(x)$ equals

$$
\frac{\Gamma(n+a+1) 2^{a-1 / 2} e^{x / 2}}{n ! \nu^{(a-1) / 2} x^{(a+1) / 2}}\left(\frac{\psi}{\psi^{\prime}}\right)^{1 / 2}\left[J_{a}(\nu \psi)+O\left(\frac{x^{1 / 2}}{\nu^{3 / 2}} \tilde{J}_{\alpha}(\nu \psi)\right)\right] \text {. }
$$

(2.10) Let $a>-1$ and $a>0$. There exists $n_{0}$ such that if $n \geq n_{0}$ and $x \geq$ $a \nu$, then $L_{n}^{\alpha}(x)$ equals

$$
\frac{(-1)^{n} \pi^{1 / 2} 2^{5 / 6} N^{N+1 / 6} e^{x / 2}}{n ! x^{(a+1) / 2} e^{N}\left[-\phi^{\prime}\right]^{1 / 2}}\left[A i\left(-\nu^{2 / 3} \phi\right)+O\left(\frac{\widetilde{A i}\left(-\nu^{2 / 3} \phi\right)}{x}\right)\right] \text {. }
$$

In each instance, the argument of $\psi, \psi^{\prime}, \phi, \phi^{\prime}$ is $x / \nu$, where the following notation is used throughout this paper:

$$
\begin{aligned}
& \nu=4 n+2 \alpha+2, N=\nu / 4 \text { in Laguerre case, } \\
& \psi=\psi(t)=1 / 2\left[\left(t-t^{2}\right)^{1 / 2}+\sin ^{-1} t^{1 / 2}\right], \quad 0 \leq t<1 ; \\
& \phi=\phi(t)= \begin{cases}{[3 b(t) / 2]^{2 / 3},} & 0<t \leq 1, \\
-[3 b(t) / 2]^{2 / 3}, & 1<t ;\end{cases} \\
& b(t)= \begin{cases}1 / 2 \cos ^{-1} t^{1 / 2}-1 / 2\left(t-t^{2}\right)^{1 / 2}, & 0<t \leq 1, \\
1 / 2\left(t^{2}-t\right)^{1 / 2}-1 / 2 \cosh ^{-1} t^{1 / 2}, & 1<t ;\end{cases} \\
& \tilde{J}_{a}(u)= \begin{cases}u^{\alpha}, & 0<u \leq 1, \\
u^{-1 / 2}, & 1<u\end{cases}
\end{aligned}
$$




$$
\widetilde{A i}(u)=\left\{\begin{array}{l}
A i(u), \quad u \geq 0, \\
{\left[|A i(u)|^{2}+|B i(u)|^{2}\right]^{1 / 2}, \quad u<0 .}
\end{array}\right.
$$

The principal branch of each inverse trigonometric and hyperbolic function is taken. $J_{a}$ is the Bessel function discussed in Watson [13]; $A i$ and $B i$ are the Airy integrals discussed in Jeffreys [6, pp. 508-511]. The rather artificial functions " $\tilde{a}_{a}$ " and " $\widetilde{A i}$ " are constructed to provide useful estimates of the Bessel and Airy functions near their respective zeros. These new functions equal or have the same order as the original functions, except in the vicinity of their zeros.

Examination of Laguerre estimates (2.9) and (2.10) and of the table on p. 699 of [1], where

$$
L_{n}^{a}(x)=\left(\frac{\Gamma(n+\alpha+1)}{n !}\right)^{1 / 2} e^{x / 2} x^{-a / 2} \mathfrak{Q}_{n}^{\alpha}(x),
$$

motivates the definition of the following function for $\alpha>-1$. We take $\nu$ equal to 2 if $n=0$ and $\alpha$ is negative.

$$
\begin{aligned}
M_{n}^{\alpha}(x) & =e^{x / 2} \nu^{\alpha}, \quad 0 \leq x \leq 1 / \nu \\
& =e^{x / 2} \nu^{\alpha / 2-1 / 4} x^{-\alpha / 2-1 / 4}, \quad 1 / \nu<x \leq \nu / 2 \\
& =e^{x / 2} \nu^{a / 2-1 / 4} x^{-\alpha / 2}\left(\nu^{1 / 3}+|x-\nu|\right)^{-1 / 4}, \quad v / 2<x \leq 3 v / 2, \\
& =e^{x / 2} \nu^{\alpha / 2} x^{-\alpha / 2} e^{-x / 4}, \quad 3 \nu / 2<x .
\end{aligned}
$$

By the discussion of the asymptotic behavior of $L_{n}^{\alpha}(x)$ in Askey and Wainger [1, pp. 697-700] and by the extension in Muckenhoupt [9], we can conclude that, given any $a>-1$, there exists a positive constant $C$, independent of $x$ and $n$, such that, for all integers $n \geq 0$,

$$
\left|L_{n}^{\alpha}(x)\right| \leq C M_{n}^{\alpha}(x) .
$$

In fact, to estimate $L_{n}^{a}(x)$ if $x>3 \nu / 2$, any $e^{-d x}, d<1 / 2$, can be used in place of $e^{-x / 4}$ of the last expression for $M_{n}^{\alpha}(x)$ in (2.11). From (2.12) and the definition of $M_{n}^{\alpha}(x)$, it follows that

$$
\left|L_{n-1}^{a+1}(x)\right| \leq C(n / x)^{1 / 2} M_{n}^{\alpha}(x), \text { for } x>1 / \nu .
$$

3. Expressions for the kernel, $K_{n, 1}$. An exact expression for the Cesàro kernel defined in (2.3) in terms of a differential operator is due to Campbell [2]; the technique for determining the operator is outlined by Ernst [5, pp. 86-87].

$$
K_{n, 1}(x, y)=\frac{(x-y)^{-1}}{n}\left[1+\frac{x+y}{(x-y)^{2}}+\frac{y \partial / \partial y-x \partial / \partial x}{x-y}\right] P_{n}(x, y)
$$

where $P_{n}(x, y)=(x-y) K_{n-1}(x, y)$ and $K_{n-1}(x, y)$ is defined in (2.2).

A sheer mechanical combination of the first and last summands of (3.1), together with (2.5), furnishes the alternate form, 


$$
K_{n, 1}(x, y)=\frac{1}{n}\left[\frac{x+y}{(x-y)^{3}} P_{n}(x, y)+\frac{y R_{n}(x, y)+x T_{n}(x, y)}{(x-y)^{2}}\right] \text {, }
$$

where

$$
\begin{aligned}
& P_{n}(x, y)=c_{n}\left[L_{n}^{\alpha}(x) L_{n}^{a-1}(y)-L_{n}^{\alpha-1}(x) L_{n}^{a}(y)\right], \\
& R_{n}(x, y)=c_{n}\left[L_{n}^{a-1}(x) L_{n}^{\alpha+1}(y)-L_{n}^{a}(x) L_{n}^{\alpha}(y)\right], \\
& T_{n}(x, y)=c_{n}\left[L_{n}^{a+1}(x) L_{n}^{a-1}(y)-L_{n}^{\alpha}(x) L_{n}^{\alpha}(y)\right],
\end{aligned}
$$

with $c_{n}=n ! / \Gamma(n+\alpha)$.

It is preferable to write an expression for $K_{n, 1}$ in terms of two Laguerre polynomials with similar estimates; namely, in terms of $L_{n}^{\alpha}(x)$ and $L_{n-1}^{\alpha+1}(x)$. This new form will lead to a kernel estimate which is significantly smaller than the obvious estimate of (3.2) and which is necessary for the proof of our main theorem.

Lemma 1. For $x$ unequal to $y$,

$$
\begin{aligned}
K_{n, 1}(x, y)= & \frac{-n !}{(x-y)^{2} \Gamma(n+\alpha+1)} \\
& \cdot\left\{(x+y) L_{n}^{a}(x) L_{n}^{a}(y)-\frac{x}{n}\left[\alpha+\frac{x+y}{x-y}-y\right] L_{n-1}^{a+1}(x) L_{n}^{a}(y)\right. \\
& \left.-\frac{y}{n}\left[x-\frac{x+y}{x-y}-x\right] L_{n-1}^{a+1}(y) L_{n}^{a}(x)+\frac{2 x y}{n} L_{n-1}^{a+1}(x) L_{n-1}^{a+1}(y)\right\} .
\end{aligned}
$$

The proof of Lemma 1 involves the substitutions of (2.6) and (2.7) into (3.2). Algebraic combination of the terms produces the form (3.3).

4. Estimates of the kernel, $K_{n, 1}$. The derivations of various estimates of the Cesàro kernel, $K_{n, 1}(x, y)$, which will be needed for the proof of Theorem 1 in $\S 5$, are given here. Theorem 1 states the main result on mean Cesàro summability of Laguerre series.

Lemma 2. If $x>0, y>0$, and $|x-y| \geq \nu / 4$, then there is a constant $C$, independent of $x, y$, and $n$, such that

$$
\left|K_{n, 1}(x, y)\right| \leq C \nu^{-(a+1)} M_{n}^{\alpha}(x) M_{n}^{a}(y) .
$$

To verify (4.1), apply estimates (2.12) and (2.13) to the kernel expression in (3.3) whenever both $x$ and $y$ are bounded above by $3 \nu / 2$. The result is immediate. If $x>3 \nu / 2$, the comment following (2.12) guarantees that $\left|L_{n}^{a}(x)\right| \leq$ $C e^{-x / 8} M_{n}^{a}(x)$. This estimate together with its analogue for $L_{n-1}^{a+1}(x)$ applied to (3.3) leads to (4.1) when one or both variables exceed $3 \nu / 2$.

Lemma 3. If $0<x, y \leq \nu / 2,1 / \nu<|x-y|<\nu / 4$, and one of the variables exceeds twice the other, then there is a constant $C$, independent of $x, y$, and $n$, sucb that 


$$
\left|K_{n, 1}(x, y)\right| \leq \frac{C \nu^{-\alpha}}{\max (x, y)} M_{n}^{\alpha}(x) M_{n}^{\alpha}(y)
$$

The assumptions of Lemma 3 together with (2.12) and (2.13) applied to (3.3) and the fact that $|x-y|>x / 2$ (or $|x-y|>y / 2$ ) immediately lead to (4.2).

In order to estimate certain integrals of the Cesàro sums of our Laguerre series in the remaining ranges, three different estimations of the kernel $K_{n, 1}$ will be needed. The first estimate will be found directly from definition (2.3); the second, from (3.3) when $x$ and $y$ range from $1 / 2 \nu$ to $3 \nu / 4$; and the third, from the fact that $L_{n-1}^{a+1}(x)$ behaves much like $-(n / x)^{1 / 2} L_{n}^{a}(x)$, for $x>1 / \nu$.

The following versions of (2.12) are included for convenient reference. Assume $\nu_{k}=4 k+2 \alpha+2$. There is a constant $C$, independent of $k$ and $x$, such that

$$
\begin{aligned}
& \left|L_{k}^{a}(x)\right| \leq C \nu_{k}^{a} e^{x / 2}, \quad 0<x \leq 1 / \nu_{k} \\
& \left|L_{k}^{a}(x)\right| \leq C \nu_{k}^{a / 2-1 / 4} e^{x / 2} x^{-\alpha / 2-1 / 4}, \quad 1 / \nu_{k}<x \leq \nu_{k} / 2 \\
& \left|L_{k}^{a}(x)\right| \leq C \nu_{k}^{a / 2} e^{x / 2} x^{-a / 2-1 / 4}\left(\nu_{k}^{1 / 3}+\left|\nu_{k}-x\right|\right)^{-1 / 4}, \quad 1 \leq x<\infty
\end{aligned}
$$

Lemma 4. For $1 / \nu \leq x, y<2 \nu$, and $|x-y|<((x+y) / n)^{1 / 2}$, there is a constant $C$, independent of both variables and $n$, such that

$$
\left|K_{n, 1}(x, y)\right| \leq C e^{(x+y) / 2}(x y)^{-\alpha / 2-1 / 4} \nu^{1 / 2} .
$$

This lemma is proved directly from the definition that

$$
K_{n, 1}(x, y)=\frac{1}{n} \sum_{k=0}^{n-1}\left(\sum_{j=0}^{k} \frac{j !}{\Gamma(j+\alpha+1)} L_{j}^{\alpha}(x) L_{j}^{a}(y)\right) \text {. }
$$

Since the expression is symmetric in $x$ and $y$, we consider only one case: $y<x$. The nature of (4.3)-(4.5) demands that the range of validity be split at 1 and the proof be done in two parts.

In general,

$$
\left|K_{n, 1}(x, y)\right| \leq \frac{C}{n} \sum_{k=0}^{n-1} \frac{n-k}{k^{a}}\left|L_{k}^{a}(x) L_{k}^{a}(y)\right| .
$$

If $1 / \nu \leq y<x<1$, the range of summation in (4.8) is separated as follows: for $k$ satisfying $1 / y \leq k \leq n-1$, (4.4) is used to estimate both Laguerre polynomials; the sum over $1 / x \leq k<1 / y$ employs (4.4) to estimate $L_{n}^{a}(x)$ and (4.3) to estimate $L_{n}^{a}(y)$; for $k$ satisfying $0 \leq k \leq 1 / y$, (4.3) is used to estimate both Laguerre polynomials. Finally, $K_{n, 1}$ is bounded by a constant multiple of

$$
e^{(x+y) / 2}\left[(x y)^{-a / 2-1 / 4} \nu^{1 / 2}+(x y)^{-a / 2-1 / 4} x^{-1 / 2}+y^{-(\alpha+1)}\right],
$$


and the first term is dominant, giving (4.6).

If $1 / 2 \leq y<x<2 \nu$, estimate (4.5) is used for both polynomials in (4.8). Simplification and use of the Schwarz inequality on

$$
\sum_{k=0}^{n-1}\left(\nu_{k}^{1 / 3}+\left|\nu_{k}-x\right|\right)^{-1 / 4}\left(\nu_{k}^{1 / 3}+\left|\nu_{k}-y\right|\right)^{-1 / 4}
$$

leave us with the estimation of

$$
\sum_{k=0}^{n-1}\left(\nu_{k}^{1 / 3}+\left|\nu_{k}-x\right|\right)^{-1 / 2}
$$

and its analogue in $y$. Replace $\nu_{k}^{1 / 3}$ by 1 and observe the sum is bounded by

$$
\sum_{\left|\nu_{k}-x\right| \leq 2 \nu}\left(1+\left|\nu_{k}-x\right|\right)^{-1 / 2} \leq C \sum_{j=1}^{2 \nu+1} j^{-1 / 2} \leq C \nu^{1 / 2} .
$$

Then, inequality (4.6) is immediate.

This completes the proof of Lemma 4 .

Lemma 5. Let $a$ be a fixed real number and $\alpha>-1$. If $1 / 2 \nu \leq x, y \leq 3 \nu / 4$ and $|x-y|>((x+y) / n)^{1 / 2}$, then there exists a constant $C$, independent of $x, y$, and $n$, such that

$$
\left|K_{n, 1}(x, y)\right| \leq \frac{C e^{(x+y) / 2}(x y)^{-a / 2-1 / 4}(x+y)}{n^{1 / 2(x-y)^{2}}} .
$$

To establish (4.9), replace $L_{n}^{\alpha}$ and $L_{n-1}^{a+1}$ in the preferred form (3.3) of the kernel by their appropriate estimates in (2.12) and (2.13).

The proof of the next lemma will require the use of the function

$$
D(x)=(n / x)^{1 / 2} L_{n}^{a}(x)+L_{n-1}^{a+1}(x) .
$$

In the range of interest, $\nu / 4 \leq x \leq 7 \nu / 4$, there is a constant $C$, independent of $n$ and $x$, such that

$$
D(x) \leq C\left(\left(|\nu-x|+\nu^{1 / 3}\right) / \nu\right)^{1 / 2} M_{n}^{a}(x)
$$

To verify (4.11), we use a procedure similar to that used to estimate the first difference, $\mathscr{L}_{n}^{a}(x)-\mathscr{L}_{n+2}^{a}(x)$, in Askey and Wainger [1]. Estimating each summand of $D(x)$ by $(2.10)$, noting $n^{-1 / 2} N^{1 / 2}$ and $e^{-1 / 2}(1-1 / 2 N)^{N-1 / 3}$ are each $1+O(1 / n)$ for $N=\nu / 4$, and observing that the error term is smaller than the principal term by a factor of $\nu^{-1}$, show $D(x)$ has the following principal term:

times

$$
(-1)^{n} \pi^{1 / 2} 2^{5 / 6} N^{N-1 / 3} x^{-\alpha / 2-1} e^{(2 x-\nu) / 4}[(n-1) !]^{-1}
$$




$$
\frac{A i\left(-\nu^{2 / 3} \phi(x / \nu)\right)}{\left[-\phi^{\prime}(x / \nu)\right]^{1 / 2}}-\frac{A i\left(-(\nu-2)^{2 / 3} \phi(x /(\nu-2))\right)}{\left[-\phi^{\prime}(x /(\nu-2))\right]^{1 / 2}} \text {. }
$$

The form of (4.12) prompts an investigation of $A i^{\prime}(z)$; from [4, p. 247], $A i^{\prime}(z)=O\left[\left(1+|z|^{1 / 2}\right) \widetilde{A i}(z)\right]$. This fact, along with the estimates

$$
\begin{aligned}
-\nu^{2 / 3} \phi(x / \nu)+(\nu-2)^{2 / 3} \phi(x /(\nu-2)) & =O\left(\phi(x / \nu)+\nu^{-1 / 3}\right), \\
{\left[\phi^{\prime}(x /(\nu-2))\right]^{-1 / 2} } & =\left[\phi^{\prime}(x / \nu)\right]^{-1 / 2}+O\left(\nu^{-1}\right),
\end{aligned}
$$

where $\phi(t)=O(|1-t|)$ and $\phi^{\prime}(t)=O(1)$, leads to the estimate given in (4.11).

Prepared with the estimate of $D(x)$, we now determine a new bound on the kernel.

Lemma 6. Let $a$ be a fixed real number and $\alpha>-1$. If $\nu / 4 \leq x, y \leq 7 \nu / 4$ and $|x-y|>1$, then there is a constant $C$, independent of $x, y$, and $n$, such that

$$
\left|K_{n, 1}(x, y)\right| \leq \frac{C \nu^{-a} e^{(x+y) / 2}}{(x-y)^{3 / 2}} .
$$

To prove Lemma 6, begin by replacing $L_{n-1}^{a+1}(x)$ in (3.3) with $D(x)-$ $(n / x)^{1 / 2} L_{n}^{a}(x)$. Combination of terms when $|x-y|>1$ and both $x$ and $y$ lie between $\nu / 4$ and $7 \nu / 4$ yields a constant $C$, independent of $x, y$, and $n$, so that

$$
\begin{aligned}
\left|K_{n, 1}(x, y)\right| \leq \frac{C \nu^{-a}}{(x-y)^{2}} & \left\{(|\nu-y|+|\nu-x|+1)\left|L_{n}^{a}(x) L_{n}^{a}(y)\right|\right. \\
& \left.+\nu\left(\left|D(x) L_{n}^{a}(y)\right|+\left|D(y) L_{n}^{a}(x)\right|+|D(x) D(y)|\right)\right\} .
\end{aligned}
$$

In the first term of the right side of (4.14), replace the $L_{n}^{a}$ functions by estimate (2.12) and note that $\nu^{1 / 2}\left(\nu^{1 / 3}+|\nu-x|\right)^{1 / 4} \geq\left(\nu^{1 / 3}+|\nu-x|\right)^{3 / 4}$ (symmetrically in $y$ ). Then, this first summand is bounded above by

$$
\frac{C \nu^{-a} e^{(x+y) / 2}}{(x-y)^{2}}\left\{\left(\frac{\nu^{1 / 3}+|\nu-x|}{\nu^{1 / 3}+|\nu-y|}\right)^{1 / 4}+\left(\frac{\nu^{1 / 3}+|\nu-y|}{\nu^{1 / 3}+|\nu-x|}\right)^{1 / 4}\right\} \text {. }
$$

Estimate (4.11) for $D(x)$ shows that the remaining term of the right side of (4.14) is smaller than (4.15) and hence can be disregarded.

Finally to arrive at the conclusion of Lemma 6 , let

$$
m=\left(\frac{\nu^{1 / 3}+|\nu-x|}{\nu^{1 / 3}+|\nu-y|}\right)^{1 / 4} \text {. }
$$

Then

$$
m \leq\left(1+\frac{|x-y|}{\nu^{1 / 3}+|\nu-y|}\right)^{1 / 4} \leq 2(x-y)^{1 / 2} \text {. }
$$


Since $|x-y|>1$, the conclusion (4.13) is immediate.

This completes the proof of Lemma 6.

5. Sufficiency theorems for Laguerre series. In this section, the conditions sufficient for mean Cesàro summability of Laguerre series will be presented and proved. The reasons for selecting the weight function, $W(x)$, and the consideration of why the conditions of Theorem 1 are also necessary appear in $\$ \$ 6,7$ and 8 , 9 , respectively.

Theorem 1. Assume $1 \leq p \leq \infty, a>-1$,

$$
W(x)=e^{-x / 2} x^{a / 2}(x /(1+x))^{a}(1+x)^{b},
$$

and

$$
\begin{aligned}
-\frac{1}{p}-\min \left(\frac{\alpha}{2}, \frac{1}{4}\right)<a<1-\frac{1}{p}+\min \left(\frac{\alpha}{2}, \frac{1}{4}\right) \\
b \leq-\frac{1}{p}+\frac{7}{4} \quad \text { if } 1 \leq p \leq 4, \\
b \leq-\frac{1}{3 p}+\frac{19}{12} \quad \text { if } 4<p \leq \infty ; \\
b \geq-\frac{1}{3 p}-\frac{5}{4} \quad \text { if } 1 \leq p<\frac{4}{3}, \\
b \geq-\frac{1}{p}-\frac{3}{4} \quad \text { if } \frac{4}{3} \leq p \leq \infty ; \\
a+b \leq-\frac{2}{p}+\frac{5}{2} \quad \text { if } 1 \leq p \leq 4, \\
a+b \leq-\frac{4}{3 p}+\frac{7}{3} \quad \text { if } 4<p \leq \infty ; \\
a+b \geq-\frac{4}{3 p}-1 \quad \text { if } 1 \leq p<\frac{4}{3} \\
a+b \geq-\frac{2}{p}-\frac{1}{2} \quad \text { if } \frac{4}{3} \leq p \leq \infty .
\end{aligned}
$$

Equality cannot occur in the first parts of (5.2) and (5.4) if $p=4$ or $a=3 / 4-$ $1 / p$ unless $p=1$. Equality cannot occur in the second parts of (5.3) and (5.5) if $p=4 / 3$ or $a=1 / 4-1 / p$ unless $p=\infty$. Then there exists a constant $C$, independent of $f(x)$ and $n$, such that

$$
\left\|\sigma_{n}(f, x) W(x)\right\|_{p} \leq C\|f(x) W(x)\|_{p},
$$

where $\sigma_{n}(f, x)$ is defined by $(2.4)$ and \|\|$_{p}$ denotes the usual norm on $(0, \infty)$.

If $p=\infty$, the usual norm convention is followed. The expressions for $p=\infty$ will be slightly different from those for finite $p$ which are presented here, but they follow similar procedures. 
We remark that for $\alpha>1 / 2$ the conditions on " $a+b$ ", (5.4) and (5.5), are each one-half unit more restrictive than those implied by summing the individual conditions on $a$ and $b$.

Taking $a=b$ in the weight function of Theorem 1 leads directly to the following corollary.

Corollary. Assume $1 \leq p \leq \infty, a>-1, W(x)=e^{-x / 2} x^{a / 2} x^{r}$,

$$
-\frac{1}{p}-\min \left(\frac{\alpha}{2}, \frac{1}{4}\right)<r<1-\frac{1}{p}+\min \left(\frac{\alpha}{2}, \frac{1}{4}\right),
$$

and $-2 / 3 p-1 / 2 \leq r \leq-2 / 3 p+7 / 6$. Then there exists some constant $C$, independent of $f(x)$ and $n$, such that

$$
\left\|\sigma_{n}(f, x) W(x)\right\|_{p} \leq C\|f(x) W(x)\|_{p},
$$

where $\sigma_{n}(f, x)$ is defined by (2.4) and \|\|$_{p}$ denotes the usual norm on $(0, \infty)$.

Theorem 2. If the bypotheses of Theorem 1 are satisfied, $p<\infty$, and $f(x) W(x)$ $\in L^{p}(0, \infty)$, then

$$
\lim _{n \rightarrow \infty} \int_{0}^{\infty}\left(\left|\sigma_{n}(f, x)-f(x)\right| W(x)\right)^{p} d x=0 .
$$

To prove Theorems 1 and 2, one might be tempted to split the intervals of integration according to the ranges of (2.11) and thereby estimate the integrand. This process would lead to the investigation of many integral pieces; for instance, 38 integrals were needed in the partial sum case [1, p. 703].

A notably simpler procedure which judiciously splits the function $f$ significantly reduces the number of cases to be investigated. The method, which was used for a similar purpose in [11, p. 449], will now be described and employed to prove Theorem 1 .

Let $f(x)=\sum_{i=1}^{4} f_{i}(x)$ where $f_{i}(x)$ agrees with $f(x)$ on $[0,1 / \nu],[1 / \nu, \nu / 2]$, $[\nu / 2,3 \nu / 2]$, and $[3 \nu / 2, \infty)$ for $i=1,2,3,4$, respectively, and each $f_{i}(x)$ is zero elsewhere. Define $E_{1}=[0,2 / \nu], E_{2}=[1 / 2 \nu, 3 \nu / 4], E_{3}=[\nu / 4,7 \nu / 4], E_{4}=$ $[5 \nu / 4, \infty)$, and $\widetilde{E}_{i}$ to be the complement of $E_{i}$ in $[0, \infty)$. This notation permits us to bound

$$
\int_{0}^{\infty}\left(\left|\sigma_{n}(f, x)\right| W(x)\right)^{p} d x
$$

with $\sigma_{n}$ defined in (2.4), by the sum of

$$
\sum_{i=1}^{4} \int_{\widetilde{E}_{i}}\left[\int_{0}^{\infty}\left|f_{i}(y) e^{-y} y^{\alpha} K_{n, 1}(x, y)\right| W(x) d y\right]^{p} d x
$$

and

$$
\sum_{i=1}^{4} \int_{E_{i}}\left|\int_{0}^{\infty} f_{i}(y) e^{-y} y^{\alpha} K_{n, 1}(x, y) W(x) d y\right|^{p} d x
$$


Our objective is then to show that (5.6) and (5.7) are each bounded above by a constant multiple of the integral $\int_{0}^{\infty}|f(x) W(x)|^{p} d x$. The parts of (5.6) fall naturally into the following categories: (1) cases where $|x-y| \geq$ $\nu / 4$, and (2) cases where $1 / \nu<|x-y|<\nu / 4$ and one of the variables $x, y$ exceeds twice the other. An appeal to the conditions of Theorem 1 is essential in dealing with (5.6). For (5.7), summands for $i=1,4$ will be treated by direct application of (2.12) to definition (4.7) of the kernel. Summands for $i=2,3$ require the use of the more delicate estimates of $K_{n, 1}$ as stated in $\$ 4$. The conditions on $a$ and $b$ in Theorem 1 will be needed only for the $E_{2}$ case. Each situation will now be examined.

For purposes of estimation, $(x /(1+x))^{a}(1+x)^{b}$ behaves like $x^{a}$ for $x<1$ and like $x^{b}$ for $x \geq 1$.

Part A. Estimation of (5.6). (1) Cases where $|x-y| \geq \nu / 4$.

These cover $x$ in $(\nu / 2, \infty)$ in the first term, $x$ in $(3 \nu / 4, \infty)$ in the second term, and all of the third and fourth terms in (5.6). Kernel estimate (4.1) is used here.

To obtain the desired bound on (5.6), piecewise evaluation demands the condition $-1 / p-\alpha / 2<a<1-1 / p+\alpha / 2$ to insure the existence of integrals on $[0,1 / \nu]$, and conditions (5.2) through (5.5). The exceptions to equality if $p=4 / 3$ or $p=4$ are also needed. To simplify the computation, analysis of estimate (4.1) and use of Hölder's in equality show $\left\|\sigma_{n}(f, x) W(x)\right\|_{p}^{p}$ is bounded by the product of

$$
\int_{0}^{\infty}\left|L_{n}^{a}(x) W(x)\right|^{p} d x\left(\int_{0}^{\infty}\left|\frac{e^{-y} y^{\alpha} L_{n}^{a}(y)}{W(y)}\right|^{q} d y\right)^{p / q}
$$

and

$$
\nu^{-(a+1) p} \int_{0}^{\infty}|f(y) W(y)|^{p} d y .
$$

By inequality (2.12) for Laguerre polynomials, the integral of (5.8) are domin ated by their values over $\left(\nu / 2, \nu-\nu^{1 / 3}\right)$. Specifically,

$$
\begin{aligned}
\int_{0}^{\infty}\left|L_{n}^{a}(x) W(x)\right|^{p} d x & \leq C \nu^{(b+a / 2-1 / 2) p+1}, \quad p<4, \\
& \leq C \nu^{(b+a / 2-1 / 3) p+1 / 3}, \quad p>4, \\
& \leq C \nu^{(b+a / 2-1 / 4) p} \log \nu, \quad p=4 ; \\
\left(\int_{0}^{\infty}\left|\frac{e^{-y} y^{a} L_{n}^{\alpha}(y)}{W(y)}\right|^{q} d y\right)^{p / q} & \leq C \nu^{(a / 2-b) p-1 / 3}, \quad p<4 / 3, \\
& \leq C \nu^{(a / 2-b+1 / 2) p-1}, \quad p>4 / 3, \\
& \leq C \nu^{(a / 2-b-1 / 4) p}(\log \nu)^{p-1}, \quad p=4 / 3 .
\end{aligned}
$$


The conditions on $b$, together with those on $a$ needed for integrals where $x<1$ or $y<1$, then imply the existence of a constant $C$, independent of $f(x)$ and $n$, such that the product of the expressions in (5.8) and $(5.8)^{\prime}$ is bounded by $C\|f(y) W(y)\|_{p}^{p}$.

This completes the proof of Case 1 , and we now turn to the second situation of Part A.

(2) Cases where $1 / \nu<|x-y|<\nu / 4$.

Two pieces of (5.6) remain:

$$
\int_{2 / \nu}^{\nu / 2}\left(\int_{0}^{1 / \nu}\left|f(y) e^{-y} y^{a} K_{n, 1}(x, y)\right| W(x) d y\right)^{p} d x
$$

and

$$
\int_{0}^{1 / 2 \nu}\left(\int_{1 / \nu}^{\nu / 2}\left|f(y) e^{-y} y^{\alpha} K_{n, 1}(x, y)\right| W(x) d y\right)^{p} d x
$$

the first is the remaining part of the first term in (5.6), the second is the remaining part of the second term in (5.6).

Since (5.9) and (5.10) are in a sense duals, the details for only (5.9) are provided here. The outer interval of integration is split at 1 to accommodate the behavior of $W(x)$. Estimate (4.2) of $K_{n, 1}$ and Hölder's in equality show that (5.9) is bounded by a constant multiple of the product of

$$
\nu^{(a / 2-1 / 4) p}\left(\int_{0}^{1 / \nu} y^{(a / 2-a) q} d y\right)^{p / q} \int_{0}^{1 / \nu}|f(y) W(y)|^{p} d y
$$

and

$$
\int_{2 / \nu}^{1} x^{(a-5 / 4) p} d x+\int_{1}^{\nu / 2} x^{(b-5 / 4) p} d x .
$$

Conditions (5.1), (5.4), and (5.5) of Theorem 1 guarantee that this product is bounded by a constant multiple of $\|f(y) W(y)\|_{p}^{p}$.

The proof of Case 2 and hence the estimation of (5.6) are completed. The proof of Theorem 1 continues with the investigation of the integrals in (5.7).

Part B. Estimation of (5.7). For both $i=1$ and $i=4$, the basic kernel expression (4.7) is most convenient for estimation.

If $i=1$ with both $x$ and $y$ in $E_{1}=[0,2 / \nu]$, then estimate (2.12) of $L_{n}^{a}$ applied to (4.7) yields $\left|K_{n, 1}(x, y)\right| \leq C \nu^{a+1}$. Then by Hölder's inequality the summand of (5.7) over $E_{1}$ is bounded by a constant multiple of $\int_{0}^{2 / \nu}|f(y) W(y)|^{p} d y$, provided $-1 / p-\alpha / 2<a<1-1 / p+\alpha / 2$ as insured by condition (5.1).

For $i=4$, the same method is employed when both $x$ and $y$ lie in $E_{4}=$ $[5 \nu / 4, \infty)$. Again, application of (2.12) to estimate the Cesàro kernel in (4.7) shows $\left|K_{n, 1}(x, y)\right| \leq C_{\nu} e^{(x+y) / 2}(x y)^{-a / 2} e^{-b(x+y)}$, where $b$ is some positive constant. With both integrals of (5.7) over $E_{4}$, use of Hölder's inequality and the fact that $\int_{5 \nu / 4}^{\infty} e^{-b x p} x^{b p} d x \leq C e^{-2 b \nu p} \nu^{b p}$ imply this piece of (5.7) is bounded by 
$C \nu e^{-r p \nu}$, where $r$ is some positive constant. The exponential guarantees the validity of the conclusion of Theorem 1 .

Cases where $i=2$ and $i=3$ demand a more precise kernel treatment. The technique involves the use of the kernel estimates (4.6) and (4.9) if $i=2$ and estimates (4.6) and (4.13) if $i=3$.

Let $i=2$. Over $E_{2}=[1 / 2 \nu, 3 \nu / 4]$, the direct estimate (4.6) of $K_{n, 1}$ is used when $|x-y|<((x+y) / n)^{1 / 2}$; the form (4.9), otherwise. In the first instance, (5.7) is majorized by

$$
\int_{E_{2}}\left|\int_{|x-y|<((x+y) / n)^{1 / 2}} \nu^{1 / 2}(x y)^{-1 / 4} \frac{x^{a, b}}{y^{a, b}} f(y) W(y) d y\right|^{p} d x .
$$

The notation $t^{a, b}$ means $t^{a}$ if $t<1$ and $t^{b}$ if $t>1$. Application of Hölder's inequality to (5.11) followed by an interchange of the order of integration yields the desired bound.

For $|x-y|>((x+y) / n)^{1 / 2}$, replace $K_{n, 1}(x, y)$ in (5.7) by its est imate (4.9). The integral is then bounded above by the sum of the next three integrals:

$$
\begin{gathered}
\int_{E_{2}}\left|\int_{1 / 4 \nu}^{x / 2} \frac{(x y)^{-1 / 4}}{n^{1 / 2} x} \frac{x^{a, b}}{y^{a, b}} f(y) W(y) d y\right|^{p} d x, \\
\int_{E_{2}}\left|\int_{3 x / 2}^{\nu} \frac{(x y)^{-1 / 4}}{n^{1 / 2} y} \frac{x^{a, b}}{y^{a, b}} f(y) W(y) d y\right|^{p} d x, \\
\int_{E_{2}}\left|\int_{1} \frac{x^{1 / 2}}{n^{1 / 2}(x-y)^{2}} f(y) W(y) d y\right|^{p} d x,
\end{gathered}
$$

where $I$ stands for $(x / \nu)^{1 / 2}<|x-y|<x / 2$.

Careful consideration of all possible ranges on $a$ and $b$ in integrals (5.12) and (5.13) is needed. Hölder's inequality and a change in the order of integration, along with the right side of (5.1), (5.2), and (5.4), show that (5.12) is bounded by a constant multiple of $\int_{0}^{\infty}|f(x) W(x)|^{p} d x$. Similarly, the same procedure, along with the left side of (5.1), (5.3), and (5.5), produces an analogous estimate for (5.13). Some exceptions to the weak inequalities on $b$ and $a+b$ as stated in Theorem 1 are also needed here. If $p=4 / 3$ or 4 , however, equality can occur.

If $p=1$, interchanging the order of integration in (5.14) and estimating lead to the desired bound. To estimate not only (5.14) but also the integral over $E_{3}$ when $p>1$, the following majorization theorem based on the Hardy maximal function is needed. The reader is referred to $[8]$ where the next theorem appears as part of Corollary 1 on p. 233.

Theorem 3. Let $m$ be an absolutely continuous finite measure on an interval $J$. Let $L(x, y)$ be a nonnegative function, monotone increasing in $y$ for $y \leq x$, 
monotone decreasing in $y$ for $y \geq x$, and $\int_{J} L(x, y) d m(y) \leq B$, where $B$ is independent of $x$. If $p>1, f \in L^{p}(d m)$, and $g(x)=\int_{J} L(x, y) f(y) d m(y)$, then $\|g\|_{p} \leq$ $B A_{p}\|f\|_{p}$, where $A_{p}$ depends only on $p$.

To apply Theorem 3 to integral (5.14), define the appropriate monotonic kernel $L(x, y)$ to be $n^{1 / 2} x^{-1 / 2}$ when $|x-y|<(x / n)^{1 / 2}$ and $x^{1 / 2} n^{-1 / 2}(x-y)^{-2}$ when $|x-y|>(x / n)^{1 / 2}$. Then over $|x-y|<(x / n)^{1 / 2}$ and $I$ of (5.14), the integral of $L(x, y)$ is bounded by 4 . Hence, Theorem 3 is applicable and asserts that (5.14) is bounded by a suitable constant multiple of $\|f(y) W(y)\|_{p}^{p}$.

It remains to examine only the $E_{3}=[\nu / 4,7 \nu / 4]$ summand of (5.7). Here the direct estimate (4.6) of $K_{n, 1}$ reduces to $C e^{(x+y) / 2} \nu^{-a}$. Comparison of same with (4.13) shows (4.6) should be used when $|x-y|<1$ and (4.13) when $|x-y|$ $>1$. Rather than treat the cases below and above 1 separately, we can combine them neatly into one term. Letting $S(x, y)$ stand for $0<|x-y|<\nu / 4$, this final piece of (5.7) is then majorized by a constant multiple of

$$
\int_{E_{3}}\left|\int_{S(x, y)} \frac{f(y) W(y)}{1+|x-y|^{3 / 2}} d y\right|^{p} d x .
$$

If $p=1$, an interchange in the order of integration leads to the required bound. If $p>1$, Theorem 3 immediately produces the conclusion of Theorem 1 , since $\left[1+|x-y|^{3 / 2}\right\rceil^{-1}$ is a suitable monotonic function.

The proof of Theorem 1 is now completed.

We next turn to the proof of Theorem 2. In order to deduce summability results of the form $\lim _{n \rightarrow \infty}\left\|\left[\sigma_{n}(f, x)-f(x)\right] W(x)\right\|_{p}=0$ from inequalities of the form $\left\|\sigma_{n}(f, x) W(x)\right\|_{p} \leq C\|f(x) W(x)\|_{p}$ as shown in Theorem 1 , standard density results for $p<\infty$ are necessary. By an argument akin to those in [12, pp. 108-110], it can be shown [11, Lemma 1, p. 437] that the functions $e^{-x / 2} x^{a}(1+x)^{b} x^{n}, n=$ $0,1,2, \cdots$, are dense in $L^{p}(0, \infty)$ for $1 \leq p<\infty$ and $a, b$ real.

To prove Theorem 2, let $\epsilon>0$ and a function $f(x)$ be given with $f(x) W(x) \epsilon$ $L^{p}(0, \infty)$ for $W(x)=e^{-x / 2} x^{a / 2}(x /(1+x))^{a}(1+x)^{b}$. Assume $C$ represents the constant of Theorem 1. By the density result just noted, there exists a polynomial $g(x)$ such that

$$
\|[f(x)-g(x)] W(x)\|_{p}<\epsilon / 2(1+C) .
$$

In combination with the conclusion of Theorem 1, this inequality yields

$$
\left\|\left[\sigma_{n}(f, x)-\sigma_{n}(g, x)\right] W(x)\right\|_{p} \leq C\|[f(x)-g(x)] W(x)\|_{p}<C \epsilon / 2(1+C) .
$$

If $r$ is the degree of $g(x)$, then, for $n>r, s_{n}(g, x)=g(x)$ and $\sigma_{n}(g, x)=$ $(p(x)+(n-r) g(x)) / n$, where $p(x)=\sum_{i=0}^{r-1} s_{i}(g, x)$ is a polynomial of degree $r-1$ with $s_{i}(g, x)$ being the $i$ th partial sum of the Laguerre series for $g$. Consequently, we find 


$$
\sigma_{n}(g, x)-g(x)=(p(x)-r g(x)) / n
$$

and for sufficiently large $n$,

$$
\left\|\left[\sigma_{n}(g, x)-g(x)\right] W(x)\right\|_{p}<C / n<\epsilon / 2 .
$$

Using the three inequalities cited and Minkowski's norm inequality for sums, we obtain for $n>r$ and $n$ greater than some $n_{0}$,

$$
\left\|\left[\sigma_{n}(f, x)-f(x)\right] W(x)\right\|_{p}<\epsilon .
$$

Thus, the proof of Theorem 2 is complete.

6. Justification of the weight function. The selection of the weight function, $W(x)$, in (1.2) is indeed not obvious. Its reasonability will be based on the following theorem.

Theorem 4. Assume $\alpha>-1, p$ is a fixed number with $1 \leq p \leq \infty$, and $q$ is defined by $1 / p+1 / q=1$. Let $\sigma_{n}(f, x)$ be the nth Cesàro mean of f's Laguerre series. Assume further that $0<W(x)<\infty$ on a set of positive measure and

$$
\int_{0}^{\infty}\left|\sigma_{n}(f, x) W(x)\right|^{p} d x \leq K \int_{0}^{\infty}|f(x) W(x)|^{p} d x,
$$

for $K$ some constant independent of $f$ and $n$. Then there exists a constant $C$, such that, for every $t \geq 1$,

$$
\begin{gathered}
\int_{1}^{t}\left|W(x) x^{-1 / 4-a / 2} e^{x / 2}\right|^{p} d x \leq C t^{3 p / 2}, \\
\int_{1 / t}^{1}\left|W(x) x^{-1 / 4-\alpha / 2} e^{x / 2}\right|^{p} d x \leq C t^{-p / 2}, \\
\int_{1}^{t}\left|W(x) x^{1 / 4-\alpha / 2} e^{x / 2}\right|^{-q} d x \leq C t^{3 q / 2}, \\
\int_{1 / t}^{1}\left|W(x) x^{1 / 4-\alpha / 2} e^{x / 2}\right|^{-q} d x \leq C t^{-q / 2} .
\end{gathered}
$$

The usual norm convention will be used if $p=\infty$ or $q=\infty$.

The Laguerre polynomials are orthogonal on $[0, \infty)$ with weight function $e^{-x} x^{a}$. To achieve the integral bounds stated in (6.2)-(6.5), the only reasonable choice for $W(x)$ is a function bounded above and below by the product of $\exp (-x / 2) x^{a / 2}$ and powers of $x$. The composition of our chosen weight function complies with this suggested form.

Lemmas 7, 8, 9 which follow are adaptations of Lemmas 1, 13, 7 which appear on pp. 423, 430, and 425 of [10], respectively. They will be needed for the proof of Theorem 4. The proof of Lemma 7 is presented here; the reader is referred to [10] for the proofs of the other lemmas. Again, the usual convention is followed if $p=\infty$ or $q=\infty$. 
Lemma 7. Assume $p$ is a fixed number satisfying $1 \leq p \leq \infty$; $\dot{\alpha}$ is a fixed number exceeding - $1 ; W(x)$ is a function for which $0<W(x)<\infty$ on a set of posi. tive measure; $f(x)$ is a function baving a Laguerre series for this a such that $\|f(x) W(x)\|_{p}<\infty$, where \|\|$_{p}$ denotes the usual unweighted norm on $(0, \infty)$. Further, let $a_{n} L_{n}^{a}(x)$ be the nth term of f's Laguerre series. Assume there exists some constant $C$, independent of $f(x)$, such that

$$
\left\|\left(a_{n} / n\right) L_{n}^{a}(x) W(x)\right\|_{p} \leq C\|f(x) W(x)\|_{p}
$$

for all $n \geq C$. Then for all $n \geq C$ and $1 / p+1 / q=1$,

$$
\left\|L_{n}^{a}(x) W(x)\right\|_{p}\left\|L_{n}^{a}(x) e^{-x} x^{a} / W(x)\right\|_{q} \leq C_{n}{ }^{\alpha+1} .
$$

To prove Lemma 7 we will show first that if $W(x)$ has the properties described in the hypotheses and $g(x) W(x) \in L^{p}(0, \infty)$, then $g(x)$ must have a Laguerre expansion. Suppose, on the contrary, there exists some $g(x)$ with $g(x) W(x) \epsilon$ $L^{p}(0, \infty)$ such that $g(x)$ has no Laguerre expansion. Let $N$ be the least nonnegative integer for which $\left\|g(x) e^{-x} x_{x}{ }^{N}\right\|_{1}=\infty$. If $N=0$, then either

(i) $\int_{0}^{\epsilon} g(x) d x=\infty$ for all $\epsilon>0$, or

(ii) $\int_{R}^{\infty} g(x) e^{-x} x a d x=\infty$ for all $R<\infty$, or

(iii) there exists an $x_{0}$ so $\int_{\left|x-x_{0}\right|<\epsilon} g(x) d x=\infty$ for all $\epsilon>0$.

In Cases (i) and (ii), for all $n$, we have $\left\|g(x) e^{-x} x_{L_{n}}^{a}(x)\right\|_{1}=\infty$ since all Laguerre polynomials have a constant term. In Case (iii), if $x_{0}$ is not a zero of $L_{n}^{a}(x)$, then $\left\|g(x) e^{-x} x^{a} L_{n}^{a}(x)\right\|_{1}=\infty$; otherwise, since $L_{n}^{a}(x)$ and $L_{n+1}^{a}(x)$ have no zeros in common, we have $\left\|g(x) e^{-x} x^{\alpha} L_{n+1}^{a}(x)\right\|_{1}=\infty$. If $N \geq 1$, then for all $n \geq N,\left\|g(x) e^{-x} x^{\alpha} L_{n}^{a}(x)\right\|_{1}=\infty$.

Let $h(x)$ be a bounded function with compact support such that $|b(x)| \leq$ $|g(x)|$. Let $b_{n} L_{n}^{a}(x)$ be the $n$th term of $b$ 's Laguerre series. Then by Hölder's inequality, for $n \geq C$,

$$
\left|b_{n}\right|\left\|L_{n}^{a}(x) W(x)\right\|_{p} \leq C\|b(x) W(x)\|_{p} \leq C\|g(x) W(x)\|_{p} .
$$

The assumption on $W(x)$ guarantees that $\left\|L_{n}^{a}(x) W(x)\right\|_{p}$ on the extreme left side of (6.7) is positive. Hence, choose $n$, with $n>N$, so that $\left\|g(x) e^{-x} x^{a} L_{n}^{a}(x)\right\|_{1}=\infty$. Then it is possible to choose $h(x)$ so $(6.7)$ is violated, thereby contradicting the assumption that $\|g(x) W(x)\|_{p}$ is finite.

Thus every function $g(x)$ with $g(x) W(x) \in L^{p}(0, \infty)$ and $W(x)$ satisfying the conditions of Lemma 7 must have a Laguerre expansion.

Using this fact, if $g(x) \in L^{p}(0, \infty)$, then $g(x) L_{n}^{a}(x) e^{-x} x^{a} / W(x)$ is in $L^{1}(0, \infty)$ for every $n$. By the converse of Hölder's inequality, for all $n$,

$$
\left\|L_{n}^{a}(x) e^{-x} x^{a} / W(x)\right\|_{q}<\infty .
$$


If $c_{n}=(n ! / \Gamma(n+\alpha+1))^{1 / 2}$, we know the coefficients $a_{n}$ of $f$ 's Laguerre series can be written as $a_{n}=c_{n}^{2} \int_{0}^{\infty} f(x) L_{n}^{a}(x) e^{-x} x^{a} d x$. By (6.8) and Hölder's inequality for a fixed $n$, there exists a function $f(x)$ such that $f(x) W(x) \in L^{p}(0, \infty)$ and

$$
\left|a_{n}\right|=c_{n}^{2}\|f(x) W(x)\|_{p}\left\|L_{n}^{a}(x) e^{-x} x^{a} / W(x)\right\|_{q} .
$$

Furthermore, by assumption, if $n \geq C$, then

$$
\left\|\left(a_{n} / n\right) L_{n}^{a}(x) W(x)\right\|_{p} \leq C\|f(x) W(x)\|_{p} .
$$

Hence, substitution of (6.9) into the left side of (6.10) and simplification produce (6.6), where by Stirling's formula $c_{n}^{2}$ is on the order of $n^{-a}$.

This completes the proof of Lemma 7.

Lemma 8. If $1 \leq p \leq \infty, a>-1$, and $0<W(x)<\infty$ on a set of positive measure, then there is a positive constant $C$ such that

$$
\left\|L_{n}^{a}(x) W(x)\right\|_{p} \geq C n^{a / 2-1 / 4}
$$

for all $n \geq 1$.

Lemm a 9. Let $W(x)$ be a measurable function, $1 \leq p \leq \infty, a>-1, \nu=4 n+$ $2 a+2$. For $y>1$, let $E_{y}=\left[5 y / 6, y-y^{1 / 2}\right]$ and let $I_{y}$ be the set integers $n$, such that $y \leq \nu \leq y+y^{1 / 3}$. Then there exists a $y_{0}$ and positive constant $C$ depending only on $p$ such that, for $y \geq y_{0}$,

$$
\left[\int_{E_{y}}\left|L_{n}^{a}(x) W(x)\right|^{p} d x\right]^{1 / p} \geq C n^{a / 2}\left[\int_{E_{y}}\left|\frac{W(x) x^{-a / 2} e^{x / 2}}{x^{1 / 4}(y-x)^{1 / 4}}\right|^{p} d x\right]^{1 / p}
$$

for at least $3 / 5$ of the $n$ 's in $I_{y}$.

7. Proof of Theorem 4. Some preliminary remarks will simplify the proof of Theorem 4.

Given a function $f(x)$, if $a_{n} L_{n}^{\alpha}(x)$ represents the $n$th term of $f$ 's Laguerre series; $s_{n}(f, x)=s_{n}$, the partial sum operator; and $\sigma_{n}(f, x)=\sigma_{n}$, the $(C, 1)$-means operator where $\sigma_{n}=(1 / n) \sum_{k=0}^{n-1} s_{k}$, then

$$
\left\|a_{n} L_{n}^{a}(x)\right\|_{p}=\left\|s_{n+1}-s_{n}\right\|_{p}
$$

and

$$
\left\|s_{n} / n\right\|_{p}=\left\|((n+1) / n) \sigma_{n+1}-\sigma_{n}\right\|_{p}
$$

By Minkowski's inequality, we find that $\left\|\left(a_{n} / n\right) L_{n}^{a}(x) W(x)\right\|_{p}$ is bounded above by

$$
\left\|((n+2) / n) \sigma_{n+2} W(x)\right\|_{p}+2\left\|((n+1) / n) \sigma_{n+1} W(x)\right\|_{p}+\left\|\sigma_{n} W(x)\right\|_{p} \text {. }
$$


If an inequality like (6.1) holds, then this is also bounded above by a constant multiple of $\|f(x) W(x)\|_{p}$. Hence, inequality (6.1) is also true if its left side is replaced by $\left\|\left(a_{n} / n\right) L_{n}^{a}(x) W(x)\right\|_{p}$ or by $\left\|\left(s_{n} / n\right) W(x)\right\|_{p}$.

In the case of Laguerre series, $a_{n} / n$ equals

$$
\frac{n !}{n \Gamma(n+\alpha+1)} \int_{0}^{\infty} e^{-y} y^{a} f(y) L_{n}^{a}(y) d y .
$$

By this result, the proof of Theorem 4 can proceed by investigating the individual terms divided by $n$.

Application of Lemma 7 shows that there is a constant $C$ such that

$$
\left\|L_{n}^{a}(x) W(x)\right\|_{p}\left\|L_{n}^{a}(x) x^{a} e^{-x} / W(x)\right\|_{q} \leq C n^{a+1} .
$$

By (6.11) of Lemma 8,

$$
n^{-1 / 2}\left\|L_{n}^{\alpha}(x) W(x)\right\|_{p} \geq C n^{\alpha / 2-3 / 4}
$$

and

$$
n^{-1 / 2}\left\|L_{n}^{a}(x) x^{a} e^{-x} / W(x)\right\|_{q} \geq C n^{\alpha / 2-3 / 4} .
$$

Hence, there exists a constant $C$ such that, for $n \geq 1$,

$$
n^{-1 / 2}\left\|L_{n}^{a}(x) W(x)\right\|_{p} \leq C n^{\alpha / 2+3 / 4}
$$

and

$$
n^{-1 / 2}\left\|L_{n}^{a}(x) x^{a} e^{-x} / W(x)\right\|_{q} \leq C n^{a / 2+3 / 4} .
$$

Using (7.2), we shall verify (6.2) and (6.3).

Define $E_{s}=[5 s / 6,11 s / 12]$. By Lemma 9, we assert the existence of constants $s_{0}$ and $C>0$, such that if $s \geq s_{0}$, then there is an $n, s / 5 \leq n \leq s / 3$, and

$$
n^{-\alpha p / 2} \int_{E_{s}}\left|L_{n}^{a}(x) W(x)\right|^{p} d x \geq C \int_{E_{s}}\left|s^{-1 / 2} w(x)\right|^{p} d x
$$

where $w(x)=x^{-\alpha / 2} e^{x / 2} W(x)$.

Then by (7.2) and the proximity of $x$ and $n$, the inequality (7.4) becomes

$$
\int_{E_{s}}\left|w(x) x^{-5 / 4}\right|^{p} d x \leq C s^{p / 2} .
$$

Now (6.2) and (6.3) will be derived from (7.5) by verifying, respectively, that

$$
\int_{1}^{t}\left|w(x) x^{-5 / 4}\right|^{p} d x \leq C t^{p / 2}
$$

and

$$
\int_{1 / t}^{1}\left|w(x) x^{3 / 4}\right|^{p} d x \leq C t^{p / 2}
$$


The validity of (7.2) for $n \geq 1$ implies $|w(x)|^{p}$ is integrable on $\left[1, s_{0}\right]$; an alteration of $C$ shows (7.6) is true for all $t, 1 \leq t \leq s_{0}$. But then to extend the validity of (7.6) to all $t \geq 1$, we add (7.6) for $t=s_{0}$ to inequalities (7.5) for suitable values of $s$.

Proof of (7.7) appeals to a commonly used formula for $L_{n}^{a}(x)$ [3, vol. 2, (7), p. 188],

$$
L_{n}^{a}(x)=\sum_{k=0}^{n}\left(\begin{array}{l}
n+a \\
n-k
\end{array}\right) \frac{(-x)^{k}}{k !} .
$$

If $0 \leq x \leq(1+\alpha) / 3 n$, then

and

$$
\left(\begin{array}{c}
n+\alpha \\
n
\end{array}\right)=\frac{\Gamma(n+\alpha+1)}{n ! \Gamma(\alpha+1)}>2 \sum_{k=1}^{n}\left(\begin{array}{l}
n+\alpha \\
n-k
\end{array}\right) \frac{(-x)^{k}}{k !}
$$

$$
L_{n}^{\alpha}(x) \geq \frac{1}{2}\left(\begin{array}{c}
n+\alpha \\
n
\end{array}\right)
$$

So there exists a positive constant $C$ relying only on $\alpha$ such that, for $n \geq 1$,

$$
L_{n}^{a}(x) \geq C n^{a}, \quad 0 \leq x \leq(1+\alpha) / 3 n .
$$

Here $\Gamma(n+\alpha+1) / n ! \geq C n^{a}$ by Stirling's formula for gamma functions.

Restricting the integration in (7.2) to the set $G_{n}=[(1+\alpha) / 6 n,(1+\alpha) / 3 n]$ and applying (7.8) yield, for $n \geq 1$,

$$
\int_{G_{n}}\left|w(x) x^{3 / 4}\right|^{p} d x \leq C n^{p / 2}
$$

Then there exists an $r_{0}$ such that, if $s \geq r_{0}$, then

$$
\int_{1 / s}^{2 / s}\left|w(x) x^{3 / 4}\right|^{p} d x \leq C s^{p / 2} \text {. }
$$

Finally, the integrability of $|w(x)|^{p}$ on $\left[1 / r_{0}, 1\right]$ implies (7.7) is true, with a different constant $C$, for all $t, 1 \leq t \leq r_{0}$. We then add (7.7) for $t=r_{0}$ to inequalities of the form (7.9) for appropriate values of $s$. The totality of these steps proves (7.7).

The proofs of (6.4) and (6.5) follow the same procedure as that of the previous parts. Using (7.3) and the analogue of (7.4), namely

$$
n^{-\alpha q / 2} \int_{E_{s}}\left|L_{n}^{a}(x) x^{a} e^{-x} / W(x)\right|^{q} d x \geq C \int_{E_{s}}\left|s^{-1 / 2 / w}(x)\right|^{q} d x,
$$

for $w(x)=x^{-\alpha / 2} e^{x / 2} W(x)$, leads directly to the desired conclusion.

8. Necessity of the summability conditions. The purpose of this and the next section is to prove that the sufficiency conditions stated in Theorem 1 are also necessary for mean Cesàro summability of Laguerre series. 
Theorem 5. If any of the inequalities (5.1)-(5.5) or the equality restrictions of Theorem 1 for $a$ and $b$ fails to bold, then there is a function $f$ for which the conclusions of Theorems 1 and 2 of $\$ 5$ are false.

The proof of this result requires several precise Laguerre polynomial estimates and integral approximations. These are furnished in this section. Weak inequalities for the conditions on $b$ and $a+b$ and the special situations when $p=4 / 3$ and $p=4$ are easily derived from appropriate norm inequalities; verification that equality cannot hold in certain other cases involves a more delicate argument. The fact that $-1 / p-\alpha / 2<a<1-1 / p+a / 2$ follows from the known existence of an integral. The inequality for $a$ with $\alpha / 2$ replaced by $1 / 4$ demands precise counterexamples. The details of the proof are presented in $\$ 9$.

In order to obtain lower bounds on certain integrals needed for the proof of our necessity theorem, several estimates of $L_{n}^{a}(x)$ are given here. These are followed by a lemma useful in determining the required integral estimates. The derivation of the Laguerre polynomial expressions (8.3) and (8.4) can be found on PP. 453-454 of [11].

Essential to the estimates for $L_{n}^{\alpha}(x)$ are the functions:

$$
d(n, \alpha, x)=(n ! / \Gamma(n+\alpha+1))^{-1 / 2} e^{x / 2} x^{-\alpha / 2},
$$

and

$$
\xi(\nu, x)=-1 / 2 \nu \cos ^{-1}\left(x^{1 / 2} \nu^{-1 / 2}\right)+1 / 2 x^{1 / 2}(\nu-x)^{1 / 2}+1 / 4 \pi .
$$

For simplicity, the arguments of $d$ and $g$ will be suppressed. Then,

$$
L_{n}^{a}(x)=\frac{d(2 / \pi)^{1 / 2}}{(\nu x)^{1 / 4}}\left[\cos \left[(\nu x)^{1 / 2}-\frac{\alpha \pi}{2}-\frac{\pi}{4}\right]+O\left(\nu^{-1 / 2} x^{-1 / 2}\right)\right],
$$

and

$$
L_{n}^{a}(x)=\frac{d(2 / \pi)^{1 / 2}(-1)^{n}}{x^{1 / 4}(\nu-x)^{1 / 4}} \cos g+O\left[\frac{1}{(\nu x)^{3 / 4}}+\frac{\nu^{1 / 4}}{(\nu-x)^{7 / 4}}\right],
$$

$$
\text { if } 1 \leq x \leq \nu-\nu^{1 / 3} \text {. }
$$

The following lemma will be useful in finding lower bounds for various integrals.

Lem ma 10. Given the expression (8.3) or (8.4), let $F(x)$ be the principal term, $h(x)$ be the argument and $k(x)$ be the coefficient of the trigonometric func. tion in $F(x)$, and $[s, t]$ be a subinterval of the stated interval of validity where $b(t)-b(s)=2 \pi$. There exists a constant $K$, independent of $s$ and $t$, such that if $t \geq K s, 1 \leq p \leq \infty$, and $r$ real, then there is some $C>0$, depending only on $r$ and $p$, such that 


$$
\int_{s}^{t}\left|F(x) x^{r}\right|^{p} d x \geq C \int_{s}^{t}\left|k(x) x^{r}\right|^{p} d x
$$

Lemma 10 is a consolidation of Lemmas 14 and 15 discussed on pp. 454-456 of [11]. If $p=\infty$, the conclusion of Lemma 10 is to be interpreted with respect to the ordinary $L^{\infty}$-norm. Although the $p=\infty$ case was not included in Lemma 15 of [11], its proof follows by a trivial observation.

9. Proof that conditions are best possible. To prove Theorem 5 , it will be shown that if the conclusion of Theorem 1 is true, then the hypotheses of Theorem 1 must hold. As a consequence of the conclusion of Theorem 1 in $\$ 5$, the justification of the chosen weight function in $\$ \$ 6$ and 7 pointed out the following results:

$$
\begin{aligned}
n^{-(\alpha+1)}\left\|L_{n}^{a}(x) W(x)\right\|_{p}\left\|L_{n}^{a}(x) e^{-x} x^{\alpha} / W(x)\right\|_{q} & \leq C, \\
n^{-(a+1) / 2}\left\|L_{n}^{\alpha}(x) W(x)\right\|_{p} & \leq C n^{3 / 4}, \\
n^{-(a+1) / 2}\left\|L_{n}^{\alpha}(x) e^{-x} x / W(x)\right\|_{q} & \leq C n^{3 / 4} .
\end{aligned}
$$

Thus

$$
\left\|L_{n}^{a}(x) W(x)\right\|_{p} \quad \text { and } \quad\left\|L_{n}^{a}(x) e^{-x} x^{\alpha} / W(x)\right\|_{q}
$$

must exist.

It is also known that if $0 \leq x \leq \delta_{n}$ for $\delta_{n}=(a+1) / 3 n$ and $n \geq 1$, then there is a positive constant $C$ relying only on a such that $L_{n}^{a}(x) \geq C n^{a}$, and hence

$$
n^{-\alpha / 2}\left\|L_{n}^{\alpha}(x) W(x)\right\|_{p} \geq C\left\|n^{\alpha / 2} W(x)\right\|_{p} .
$$

Since the left side of this inequality is finite and $\left\|n^{\alpha / 2} W(x)\right\|_{p}^{p}$ is greater than or equal to

$$
C \int_{0}^{\delta} n\left|n^{a / 2} e^{-x / 2} x^{a / 2} x^{a}\right|^{p} d x \geq C \int_{0}^{\delta} n x^{(a / 2+a) p} d x
$$

the existence of the last integral implies $a>-1 / p-a / 2$. Similarly $\int_{0}^{\delta} n x^{(a / 2-a) q} d x$ can be shown to exist and therefore $a<1-1 / p+\alpha / 2$.

Now in order to find lower bounds for the individual norms of (9.1), Lemma 10 is applied to estimate (8.4). The computation, similar to [11, p. 457], reveals the existence of an $n_{0}$ and $C>0$, such that if $n>n_{0}$, then

$$
\begin{aligned}
n^{-(a+1) p / 2}\left\|L_{n}^{a}(x) W(x)\right\|_{p}^{p} & \geq C \nu^{(b-1) p+1}, & & 1 \leq p<4, \\
& \geq C \nu^{(b-1 / 2) p-1} \log \nu, & & p=4, \\
& \geq C \nu^{(b-5 / 6) p+1 / 3}, & & 4<p \leq \infty ;
\end{aligned}
$$

and 


$$
\begin{aligned}
& n^{-(a+1) p / 2}\left\|L_{n}^{\alpha}(x) e^{-x} x / W(x)\right\|_{q}^{p} \geq C \nu^{-(b+1 / 2) p-1 / 3}, \quad 1 \leq p<4 / 3, \\
& \geq C \nu^{-(b+3 / 2) p+1}(\log \nu)^{1 / 3}, \quad p=4 / 3, \\
& \geq C \nu^{-b p-1}, \quad 4 / 3<p \leq \infty .
\end{aligned}
$$

If $p=\infty$, the usual convention is followed.

Relating (9.2) and (9.4) produces the inequalities $b \leq-1 / p+7 / 4$ for $1 \leq$ $p<4, b<-1 / p+7 / 4$ for $p=4$, and $b \leq-1 / 3 p+19 / 12$ for $4<p \leq \infty$.. Any larger values of $b$ would contradict the stated norm inequalities. A similar relationship between (9.3) and (9.5) shows $b \geq-1 / 3 p-5 / 4$ for $1 \leq p<4 / 3$, $b>-1 / p-3 / 4$ for $p=4 / 3$ and $b \geq-1 / p-3 / 4$ for $4 / 3<p \leq \infty$. Any smaller values of $b$ would contradict the stated norm inequalities.

Again, use of Lemma 10 and estimate (8.3) gives the following lower bounds.

$$
\begin{array}{rlrl}
n^{-(a+1) p / 2}\left\|L_{n}^{\alpha}(x) W(x)\right\|_{p}^{p} & \geq C \nu^{-3 p / 4}, & & a<-1 / p+1 / 4, \\
& \geq C \nu^{-(a+1 / 2) p-1}, & a \geq-1 / p+1 / 4 .
\end{array}
$$

$$
\begin{array}{rlrl}
n^{-(a+1) p / 2}\left\|L_{n}^{a}(x) e^{-x} x a / W^{\prime}(x)\right\|_{q}^{p} & \geq C \nu^{-3 p / 4}, & a>-1 / p+3 / 4, \\
& \geq C \nu^{(a-3 / 2) p+1}, \quad a \leq-1 / p+3 / 4 .
\end{array}
$$

The product of estimates in (9.4) and (9.7) compared with (9.1) shows that $a+b \leq-2 / p+5 / 2$ for $1 \leq p<4, a+b<-2 / p+5 / 2$ for $p=4$, and $a+b$ $\leq-4 / 3 p+7 / 3$ for $4<p \leq \infty$. Analogously, the product of estimates in (9.5) and (9.6) compared with (9.1) yields the conditions $a+b \geq-4 / 3 p-1$ for $1 \leq p<4 / 3$, $a+b \geq-2 / p-1 / 2$ for $4 / 3<p \leq \infty$, and $a+b>-2 / p-1 / 2$ for $p=4 / 3$. Values outside the specified ranges of $a+b$ would violate the given norm inequalities.

Multiplication of suitable lower bounds in (9.6) and (9.7) compared with the constant bound of (9.1) shows the necessity of having $-1 / p-5 / 4 \leq a \leq-1 / p+9 / 4$. Further investigation will diminish this range of $a$.

It remains to verify the necessity of the conditions $-1 / p-1 / 4<a<5 / 4-1 / p$, $b$ cannot equal $7 / 4-1 / p$ if $a=3 / 4-1 / p$ and $1<p<4$, and $b$ cannot equal $-3 / 4-1 / p$ if $a=1 / 4-1 / p$ and $4 / 3<p<\infty$. The fact that $a+b$ cannot equal $-2 / p+5 / 2$ if $a=3 / 4-1 / p$ and $1<p<4$ nor $-2 / p-1 / 2$ if $a=1 / 4-1 / p$ and $4 / 3<p<\infty$ follows immediately from the special conditions on $b$. These conditions are not necessary to maintain (9.1).

Elimination of equality at the extremes of the ranges of $a$ and $b$ and the restriction of the range of $a$ stem from the integration in (5.7) over $E_{2}=[1 / 2 \nu$, $3 \nu / 4]$. Should equality occur for $b$ or should $-1 / p-5 / 4 \leq a \leq-1 / p-1 / 4$ or $5 / 4-1 / p \leq a \leq-1 / p+9 / 4$, we will show that there is a counterexample to the conclusion of the sufficiency theorem of $\$ 5$. 
It will be established that only the initial term of the Cesàro kernel expression, (3.3), need be considered to determine counterexamples. Specifically, the term is

$$
\left(-n !(x+y) / \Gamma(n+\alpha+1)(x-y)^{2}\right) L_{n}^{a}(x) L_{n}^{a}(y) .
$$

This will insure against any possible cancellation. For both $x$ and $y$ in $E_{2}$, the intermediate summands of $K_{n, 1}(x, y)$ used in integral (5.7) do produce the bound, $C\|f(x) W(x)\|_{p}^{p}$, for the values of $a$ and $b$ in question. Thus, they do not contribute to counterexamples.

Examination of the remaining first and last summands of $K_{n, 1}(x, y)$ in (3.3) focuses on the signs of $L_{n-1}^{a+1}(x)$ and $L_{n}^{a}(x)$ on $E_{2}$. Comparison of their respective cosine arguments from estimates (8.3) and (8.4) shows their difference never exceeds $\pi / 2$. Consequently, $L_{n-1}^{a+1}(x)$ and $L_{n}^{a}(x)$ have the same sign, on at least one-half of each period of the principal term and the opposite sign on the rest. This fact motivates the definition, for each $n$, of the function $g_{n}(y)$.

(9.9) $g_{n}(y)=e^{y / 2} y^{-\alpha / 2} y^{r}\left\{\begin{array}{l}\operatorname{sgn} L_{n}^{\alpha}(y), \quad \text { if } L_{n}^{a} \text { and } L_{n-1}^{a+1} \text { have the same sign, } \\ 0, \text { if } L_{n}^{\alpha} \text { and } L_{n-1}^{\alpha+1} \text { have opposite signs, }\end{array}\right.$ on $[1 / \nu, \nu / 2]$, and $g_{n}(y)=0$ elsewhere. Here, $r$ is some real number whose value will be specified in each counterexample.

We will then proceed to investigate the integral,

$$
\int_{E_{2}}\left|\int_{E_{2}} g_{n}(y) e^{-y} y^{a_{K}} K_{n, 1}(x, y) W(x) d y\right|^{p} d x,
$$

which is smaller than the basic integral,

$$
\int_{0}^{\infty}\left|\sigma_{n}(f, x) W(x)\right|^{p} d x
$$

If any of the remaining conditions on $a$ or $b$ is violated, it will be shown that there exists an $r$ such that (9.10) is an unbounded function of $n$ for this $g_{n}(y)$ when $K_{n, 1}$ is represented by (9.8). However, if any other term of the kernel expression is used, (9.10) is a bounded function of $n$, and the conclusion of Theorem 1 is satisfied.

To analyze each condition on $a$ and $b$, we will use Lemma 10. As written, this lemma applies only to the principal term of the Laguerre polynomial estimate. Nevertheless, a similar procedure shows the integrals of the error terms pose no problems since they are sufficiently small in each range of concern.

Suppose $a=5 / 4-1 / p$ and $K$ is the constant of Lemma 10. The source of our counterexample is integral (5.12). Choose $s$ so $s \leq 1$ and, for $n$ large enough, $2 K^{2} / \nu \leq s$ and $\int_{2 K / \nu}^{s} x^{a p} d^{-p}\left|L_{n}^{a}(x)\right|^{p} d x$ has its principal term at least twice its error term by (8.3) and Lemma 10. Here $d^{-1}$ is the reciprocal of $d(n, \alpha, x)$ defined by (8.1). If the conclusion of Theorem 1 were true for $a=5 / 4-1 / p$ and 
$r=-3 / 2$ in (9.9), then there would be a $C$, independent of $g_{n}(x)$ and $n$, such that

$$
\int_{2 K / \nu}^{s}\left|\int_{1 / \nu}^{x / 2} g_{n}(y) e^{-y} y^{a} K_{n, 1}(x, y) W(x) d y\right|^{p} d x \leq C \nu^{p / 4}
$$

The restriction of $g_{n}(y)$ to $[1 / \nu, x / 2]$ and 0 elsewhere is taken. The only term of $K_{n, 1}(x, y)$ for which (9.11) fails is the summand (9.8). With (9.8), use of Lemma 10 and (8.3) shows the left side of (9.11) is bounded below by

$$
C \nu^{-p / 2} \int_{2 K / \nu}^{s} x^{(a-5 / 4) p}\left[\int_{1 / \nu}^{x / 2} y^{r-1 / 4} d y\right]^{p} d x
$$

and hence by $C \nu^{p / 4} \log \nu$ when $r=-3 / 2$. Thus, $a$ cannot equal $5 / 4-1 / p$.

Suppose $5 / 4-1 / p<a \leq 9 / 4-1 / p$. Following the same procedure as the preceding equality case yields a similar contradiction when $r=-5 / 2$. These values of $a$ must therefore be ruled out.

Suppose $a=-1 / 4-1 / p$ and $K$ is the constant of Lemma 10. Integral (5.13) is now the source of the problem. Choose $s \leq 1$ so that for $n$ sufficiently large, $2 K^{2} / \nu \leq s$ and $3 s K / 2 \leq 1$ and the integral of $\left|d^{-1} L_{n}^{a}(y)\right|$ over $[3 x / 2,1]$ has its principal term at least twice the error term when using (8.3) and Lemma 10 to estimate it. Again $d^{-1}$ is the reciprocal of (8.1). If the conclusion of our sufficiency theorem were valid when $a=-1 / 4-1 / p$ and $r=1 / 4$, then there would be a suitable constant $C$ such that

$$
\int_{2 K / \nu}^{s}\left|\int_{3 x / 2}^{1} g_{n}(y) e^{-y} y^{\alpha} K_{n, 1}(x, y) W(x) d y\right|^{p} d x \leq C \log \nu .
$$

Again, only term (9.8) of $K_{n, 1}(x, y)$ yields a contradiction of (9.12). Assuming $g_{n}(y)$ is 0 outside $[3 x / 2,1],(9.8),(8.3)$, and Lemma 10 show the left side of (9.12) is bounded below by

$$
C \nu^{-p / 2} \int_{2 K / \nu}^{s} x^{(a-1 / 4) p}\left[\int_{3 x / 2}^{1} y^{-1} d y\right]^{p} d x
$$

This is then bounded below by $C(\log \nu)^{p}$. So a cannot equal $-1 / 4-1 / p$ unless $p=1$.

The fact that $a$ cannot equal $-1 / 4-1 / p$ even if $p=1$ follows from investigation of the left side of (9.12) with the inner interval of integration in $y$ changed to $[1, \nu / 2]$. If $r=1 / 4$ in (9.9), this integral is bounded below by $C(\log \nu)^{p}$, while the conclusion of Theorem 1 would imply a simple constant upper bound on this integral. Consideration of all possible cases eliminates any reliance on $b$.

Suppose $-5 / 4-1 / p \leq a<-1 / 4-1 / p$. Let $r=2$ in the definition (9.9) of $g_{n}(y)$. Following the same procedure as the equality case, the left side of (9.12) should be bounded above by some constant $C$ if Theorem 1 were true. However, use of (9.8) together with estimate (8.3) and Lemma 10 shows the left side is bounded below by $C \nu^{-(a+1 / 4) p-1}$, contradicting the conclusion of Theorem 1 . 
Hence, $a$ cannot assume any value less than $-1 / 4-1 / p$, since we already know that $a<-5 / 4-1 / p$ is impossible.

Suppose $b=7 / 4-1 / p$ and $a=3 / 4-1 / p$ for $1<p<4$. Let $K$ be the constant of Lemma 10 and choose $s>1$ so that for $n$ sufficiently large $2 K^{2} s \leq \nu / 2$ and the integral of $\left|d^{-1} L_{n}^{a}(x)\right|$ over $[1 / 4 \nu, 1]$ exceeds a constant multiple of $\nu^{-1 / 4}$ by Lemma 10 and (8.3). Then if $r=-3 / 4$, and $g_{n}(y)$ is 0 outside $[1 / 4 \nu, 1]$, the conclusion of Theorem 1 in $\$ 5$ guarantees a constant $C$, independent of $g_{n}(x)$ and $n$, such that

$$
\int_{2 K s}^{\nu / 2}\left|\int_{1 / 4 \nu}^{1} g_{n}(y) e^{-y} y^{\alpha} K_{n, 1}(x, y) W(x) d y\right|^{p} d x \leq C \log \nu .
$$

However, by use of (9.8), (8.3), (8.4), and Lemma 10, the left side of (9.13) is bounded below by

$$
C \nu^{-p / 2} \int_{2 K s}^{\nu / 2} x^{(b-5 / 4) p}\left[\int_{1 / 4 \nu}^{1} y^{-1} d y\right]^{p} d x
$$

which in turn is bounded below by $C(\log \nu)^{p}$. Hence, a contradiction exists whenever $p>1$ and consequently $b$ cannot equal $7 / 4-1 / p$ if $a=3 / 4-1 / p$ and $1<p<4$.

Similarly, suppose $b=-3 / 4-1 / p$ when $a=1 / 4-1 / p$ and $4 / 3<p<\infty$. Choose $s>1$ so the integral of $x^{b p} d^{-p}\left|L_{n}^{a}(x)\right|^{p}$ on $[1, \nu / 2]$ exceeds $C \nu^{-p / 4}$ for large $n$ and so $2 K^{2} / \nu \leq s$. If $r=1$ in (9.9) and $g_{n}(y)$ is suitably restricted, then Theorem 1 implies there is an appropriate $C$ such that

$$
\int_{2 K ! \nu}^{s}\left|\int_{1}^{\nu / 2} g_{n}(y) e^{-y} y^{\alpha} K_{n, 1}(x, y) W(x) d y\right|^{p} d x \leq C \nu^{p / 4} .
$$

Expression (9.8) for the kernel, (8.3) and (8.4) for the Laguerre polynomials, and Lemma 10 show the left side of (9.14) is bounded below by $C \nu^{p / 4} \log \nu$. The other terms of $K_{n, 1}$ do yield the integral bound of (9.14). Hence such equalities on $b$ and $a$ cannot occur.

Thus the proof of Theorem 5 is complete. Necessary and sufficient conditions for mean Cesàro summability of Laguerre series with the stated weight function are now established.

10. Facts about Hermite polynomials and series. The Hermite polynomials, $H_{n}(x)$, defined by

$$
\sum_{n=0}^{\infty} H_{n}(x) r^{n} / n !=\exp \left(2 x r-r^{2}\right)
$$

are orthogonal on $(-\infty, \infty)$ with weight function $e^{-x^{2}}$.

Crucial for our application of Laguerre series summability results in $\$ \$ 5$ and 8 to the Hermite series case is the ability to represent Hermite polynomials in 
terms of Laguerre polynomials with the parameters $a= \pm 1 / 2$.

$$
\begin{gathered}
H_{2 n}(x)=(-1)^{n} 2^{2 n} n ! L_{n}^{-1 / 2}\left(x^{2}\right), \\
H_{2 n+1}(x)=(-1)^{n} 2^{2 n+1} n ! x L_{n}^{1 / 2}\left(x^{2}\right) .
\end{gathered}
$$

It is well known that the Hermite series expansion of a function $f(x)$ is $\sum_{n=0}^{\infty} a_{n} H_{n}(x)$, where

$$
a_{n}=\left(\pi^{1 / 2} 2^{n} n !\right)^{-1} \int_{-\infty}^{\infty} e^{-x^{2}} f(x) H_{n}(x) d x
$$

By definition [14, Vol. I, Pp. 75-77], the $n$th $(C, 1)$ mean of this series is given by

$$
\tau_{n}(f, x)=\frac{1}{n} \sum_{k=0}^{n-1}\left[\sum_{j=0}^{k} a_{j} H_{j}(x)\right] .
$$

The kernel of the partial sums will be defined by

$$
D_{n}(x, y)=\pi^{-1 / 2} \sum_{k=0}^{n}\left(2^{k} k !\right)^{-1} H_{k}(x) H_{k}(y) .
$$

Denoting the kernel of the $(C, 1)$-means $\tau_{n}(f, x)$ by

$$
D_{n, 1}=D_{n, 1}(x, y)=\frac{1}{n} \sum_{k=0}^{n-1} D_{k}(x, y)
$$

yields the expression

$$
\tau_{n}(f, x)=\int_{-\infty}^{\infty} D_{n, 1}(x, y) e^{-y^{2}} f(y) d y .
$$

With the notation \|\|$_{p}$ representing the usual $L^{p}$-norm over $(-\infty, \infty)$, our purpose is to show inequalities of the form

$$
\left\|\tau_{n}(f, x) U(x)\right\|_{p} \leq C\|f(x) U(x)\|_{p},
$$

where $U(x)$ is the weight function $e^{-x^{2} / 2}(|x| /(1+|x|))^{A}(1+|x|)^{B}$ and $C$ is a constant which is independent of $n$ and $f(x)$. Mean Cesàro summability conclusions will be drawn in the standard manner by use of the appropriate density facts.

In this section, equivalent forms of the Hermite kernels, (10.4) and (10.5), will be stated in terms of Laguerre polynomials. The main theorems for Hermite series summability, which are given in $\$ 11$, will then follow directly from the results for the Laguerre series.

As indicated by (10.1) and (10.2), the conversion from Hermite polynomials, $H_{n}(x)$, to Laguerre polynomials depends on whether $n$ is an even or an odd integer. Consequently, to establish workable expressions for the Hermite kernels, it is advisable to separate the even and odd indices of each sum. 
We will derive the Cesàro kernel expressions for $D_{2 n, 1}(x, y)$ and $D_{2 n+1,1}(x, y)$, defined in (10.5).

Also, since the focus is now on particular values of the parameter $\alpha$, the notation $K_{n}^{a}$ and $K_{n, 1}^{a}$ is introduced for the Laguerre series partial sum and Cesàro kernels, respectively, of (2.1) and (2.3).

From the definition, the following Cesàro kernel expression for Hermite series can be written.

$$
D_{2 n, 1}(x, y)=\frac{1}{2 n} \sum_{k=0}^{n-1}\left[D_{2 k}(x, y)+D_{2 k+1}(x, y)\right] .
$$

To determine expressions for $D_{2 k}$ and $D_{2 k+1}$ in terms of Laguerre partial sum kernels, we sum the even and odd indices of (10.4) separately and replace $H_{k}(x)$ and $H_{k}(y)$ by either (10.1) or (10.2). Application of the fact that $\Gamma(z) \Gamma(z+1 / 2)$ $=2^{1-2 z} \pi^{1 / 2} \Gamma(2 z)$ from [12, (1.7.6), p. 14] simplifies the coefficient of the sum over even subscripts. Specifically, this coefficient is

$$
2^{2 k}(k !)^{2} / \pi^{1 / 2}(2 k) !=k ! / \Gamma(k+1 / 2) .
$$

Using a similar computation for the odd subscripts shows that $D_{2 k}(x, y)$ equals the sum of

$$
\sum_{j=0}^{k} \frac{j !}{\Gamma(j+1 / 2)} L_{j}^{-1 / 2}\left(x^{2}\right) L_{j}^{-1 / 2}\left(y^{2}\right)
$$

and

$$
\text { xy } \sum_{j=0}^{k-1} \frac{j !}{\Gamma(j+3 / 2)} L_{j}^{1 / 2}\left(x^{2}\right) L_{j}^{1 / 2}\left(y^{2}\right) .
$$

Comparison of these sums with the partial sum kernel, (2.1), shows that (10.8) is $K_{k}^{-1 / 2}\left(x^{2}, y^{2}\right)$ and $(10.9)$ is $x y K_{k-1}^{1 / 2}\left(x^{2}, y^{2}\right)$. As usual, $K_{k-1}^{1 / 2}$ is taken to be 0 when $k=0$.

Following the same procedure, it is apparent that

$$
D_{2 k+1}(x, y)=K_{k}^{-1 / 2}\left(x^{2}, y^{2}\right)+x y K_{k}^{1 / 2}\left(x^{2}, y^{2}\right) .
$$

In (10.7), if $D_{2 k}(x, y)$ is replaced by the Laguerre kernel form of (10.8) and (10.9) and $D_{2 k+1}(x, y)$ is replaced by (10.10), then

$$
D_{2 n, 1}(x, y)=K_{n, 1}^{-1 / 2}\left(x^{2}, y^{2}\right)+(x y / 2) K_{n, 1}^{1 / 2}\left(x^{2}, y^{2}\right)
$$

$$
+(x y(n-1) / 2 n) K_{n-1,1}^{1 / 2}\left(x^{2}, y^{2}\right) .
$$

Expression (2.3) is used to determine (10.11).

The following form is derived in the same manner as (10.11). 


$$
\begin{aligned}
D_{2 n+1,1}(x, y)= & \frac{n+1}{2 n+1} K_{n+1,1}^{-1 / 2}\left(x^{2}, y^{2}\right) \\
& +\frac{n}{2 n+1} K_{n, 1}^{-1 / 2}\left(x^{2}, y^{2}\right)+\frac{2 n}{2 n+1} x y K_{n, 1}^{1 / 2}\left(x^{2}, y^{2}\right) .
\end{aligned}
$$

Using either (10.11) or (10.12), the typical Cesàro kernel of Hermite series is explicitly represented in terms of the Laguerre series kernel. It will be proved in $\$ 11$ that the theorems about Hermite expansions are then immediate conse quences of those about Laguerre expansions.

11. Application to Hermite series. In order of presentation, the following three theorems can be treated as corollaries to Theorems 1 and 2 in $\$ 5$ and Theorem 5 in $\$ 8$.

Theorem 6. Assume $1 \leq p \leq \infty$,

$$
U(x)=e^{-x^{2} / 2}(|x| /(1+|x|))^{A}(1+|x|)^{B}, \quad-1 / p<A<-1 / p+1 ;
$$

$$
\begin{array}{ll}
B \leq-1 / p+3 & \text { if } 1 \leq p \leq 4, \\
B \leq 1 / 3 p+8 / 3 & \text { if } 4<p \leq \infty ; \\
B \geq 1 / 3 p-3 & \text { if } 1 \leq p<4 / 3, \\
B \geq-1 / p-2 & \text { if } 4 / 3 \leq p \leq \infty .
\end{array}
$$

Assume further that equality cannot occur in (11.2) if $p=4$ nor in (11.3) if $p=4 / 3$. Then there exists a constant $C$, independent of $f(x)$ and $n$, such that

$$
\left\|\tau_{n}(f, x) U(x)\right\|_{p} \leq C\|f(x) U(x)\|_{p},
$$

where $\tau_{n}(f, x)$ is defined by (10.6) and \|\|$_{p}$ denotes the usual norm on $(-\infty, \infty)$.

The ordinary norm convention is followed if $p=\infty$. We remark that the range of $A$ is extended one-half unit in each direction whenever only odd order Hermite polynomials are involved and $\alpha=1 / 2$ in (10.2). Furthermore, since the special conditions on $a+b$ given in (5.4) and (5.5) were relevant only if $a>-1 / 2$, we would not expect such conditions on $A+B$.

To prove Theorem 6, it should be observed that the kernels in (10.11) and (10.12) can be expressed entirely as a linear combination of terms like $K_{n, 1}^{-1 / 2}\left(x^{2}, y^{2}\right)$ and $x y K_{n, 1}^{1 / 2}\left(x^{2}, y^{2}\right)$. Hence, it suffices to consider only these two terms in $\tau_{2 n}(f, x)$. Using $K_{n, 1}^{-1 / 2}\left(x^{2}, y^{2}\right)$, making the change of variable, $x^{2}=r$ and $y^{2}=t$, and multiplying and dividing the integrand by $(1+|r|)^{1 / 4+1 / 2 p}$, show that the part of $\left\|\tau_{2 n}(f, x) U(x)\right\|_{p}^{p}$ resulting from this term of the kernel is bounded 
above by a constant, independent of $f(x)$ and $n$, times

$$
\int_{0}^{\infty}\left[\int_{0}^{\infty}\left|K_{n, 1}^{-1 / 2}(r, t)\right| e^{-t} t^{-1 / 2} \mathcal{T}(t) W(r) d t\right]^{p} d r
$$

where $\mathcal{T}(t)=\left|f\left(t^{1 / 2}\right)\right|+\left|f\left(-t^{1 / 2}\right)\right|$ and

$$
W(r)=e^{-r / 2} r^{-1 / 4}(r /(1+r))^{a}(1+r)^{b},
$$

for $a=A / 2+1 / 4-1 / 2 p$ and $b=B / 2+1 / 4-1 / 2 p$.

Now (11.5) is precisely in the form of the Laguerre weight function, and (11.4) is the analogue of $\left\|\sigma_{n}(f, x) W(x)\right\|_{p}^{p}$ in Theorem 1. Conditions (11.1)-(11.3) here and Theorem 1 show that (11.4) is majorized by

$$
C \int_{0}^{\infty}\left[\left(\left|f\left(t^{1 / 2}\right)\right|+\left|f\left(-t^{1 / 2}\right)\right|\right) W(t)\right]^{p} d t .
$$

Another change of variable with $t=x^{2}$ leads to the majorization of (11.6) by $C \int_{-\infty}^{\infty}|f(x) U(x)|^{p} d x$. Thus, the conclusion of Theorem 6 in the present section is satisfied. We remark that the stated conditions on $A$ and $B$ are obtained by letting $a=A / 2+1 / 4-1 / 2 p$ and $b=B / 2+1 / 4-1 / 2 p$ for $a=-1 / 2$ in (5.1)(5.3) of Theorem 1. The equality restrictions are immediate from the Laguerre case.

A similar situation arises if the term $x y K_{n, 1}^{1 / 2}\left(x^{2}, y^{2}\right)$ is considered in the Cesàro kernel. This completes the proof of Theorem 6 .

Letting $A=B$ in the weight function of Theorem 6 leads directly to the following corollary.

Corollary. Assume $1 \leq p \leq \infty, U(x)=e^{-x^{2} / 2}|x|^{r},-1 / p<r<-1 / p+1$. Then there exists some constant $C$, independent of $f(x)$ and $n$, sucb that

$$
\left\|\tau_{n}(f, x) U(x)\right\|_{p} \leq C\|f(x) U(x)\|_{p},
$$

where $\tau_{n}(f, x)$ is defined by (10.6) and \|\|$_{p}$ denotes the usual norm on $(-\infty, \infty)$.

Theorem 7. If the bypotheses of Theorem 6 are satisfied, $p<\infty$, and $f(x) U(x) \in L^{p}(-\infty, \infty)$, then

$$
\lim _{n \rightarrow \infty} \int_{-\infty}^{\infty}\left[\left|\tau_{n}(f, x)-f(x)\right| U(x)\right]^{p} d x=0
$$

The proof of Theorem 7 is based on the fact that the functions $e^{-x^{2} / 2}(1+|x|)^{B} x^{n+A}$, for $n=0,1,2, \cdots$, and $A$ and $B$ real, are dense in $L^{p}(-\infty, \infty)$ for $1 \leq p<\infty$.

Theorem 8. If any of the inequalities in (11.1)-(11.3) or the equality restrictions of Theorem 6 fails to hold, then there is a function f for which the conclusions of Theorems 6 and 7 are false.

All the conditions of Theorem 6 arose from considering $K_{n, 1}^{-1 / 2}\left(x^{2}, y^{2}\right)$ in 
the Cesàro kernel expression. If an even function $f(x)$ is used together with $D_{2 n, 1}(x, y)$ in $\left\|\tau_{2 n}(f, x) U(x)\right\|_{p}$, then a change of variable like that in the proof of Theorem 6 shows $\left\|\tau_{2 n}(f, x) U(x)\right\|_{p}^{p}$ is bounded below by a positive constant multiple of

$$
\int_{0}^{\infty}\left[\int_{0}^{\infty}\left|K_{n, 1}^{-1 / 2}(r, t)\right| e^{-t} t^{-1 / 2}\left|f\left(t^{1 / 2}\right)\right| W(r) d t\right]^{p} d r
$$

With $W(r)$ given in (11.5), it follows that (11.7) is majorized by $C \int_{0}^{\infty}\left|f\left(t^{1 / 2}\right) W(t)\right|^{p} d t$. Then Theorem 5 of $\$ 8$ is applicable, and the necessity of our conditions on $A$ and $B$ follows from the analogous situation for Laguerre series. Thus, Theorem 8 is proved.

This completes the discussion of the application of Laguerre series summability results to the Hermite series case. The necessary and sufficient conditions for mean Cesàro summability of Hermite series have therefore been determined.

\section{REFERENCES}

1. R. Askey and S. Wainger, Mean convergence of expansions in Laguerre and Hermite series, Amer. J. Math. 87 (1965), 695-708. MR 32 \#316.

2. R. Campbell, Détermination effective de toutes les moyennes de Cesàro d'ordre entier pour les séries de polynomes orthogonaux comprenant ceux de Laguerre et de Hermite, C. R. Acad. Sci. Paris 243 (1956), 882-885. MR 18, 125.

3. A. Erdélyi et al., Higher transcendental functions. Vol. II, Bateman Manuscript Project, McGraw-Hill, New York, 1953. MR 15, 419.

4. A. Erdélyi, Asymptotic forms for Laguerre polynomials, J. Indian Math. Soc. 24 (1960), 235-250. MR 23 \#A1073.

5. D. Ernst, Über die Konvergenz der $(C, 1)$-Mittel von Fourier-Laguerre-Reihen, Compositio Math. 21 (1969), 81-101. MR $39 \# 3231$.

6. H. Jeffreys and B. Swirles Jeffreys, Methods of mathematical physics, 3rd ed., Cambridge Univ. Press, Cambridge, 1956. MR 17, 590.

7. E. Kogbetliantz, Sur les moyennes arithmétiques des séries-noyaux des développements en séries $d^{\prime H}$ Hermite et de Laguerre et sur celles de ces séries-noyaux derivées terms à terme, J. Math. Phys. 14 (1935), 37-99.

8. B. Muckenhoupt, Poisson integrals for Hermite and Laguerre expansions, Trans. Amer. Math. Soc. 139 (1969), 231-242. MR 40 \#3158.

9. - - A symptotic forms for Laguerre polynomials, Proc. Amer. Math. Soc. 24 (1970), 288-292. MR $40 \# 4503$.

10. - Mean convergence of Hermite and Laguerre series. I, Trans. Amer. Math. Soc. 147 (1970), 419-431. MR $41 \# 711$.

11. - Mean convergence of Hermite and Laguerre series. II, Trans. Amer. Math. Soc. 147 (1970), 433-460. MR $41 \# 711$.

12. G. Szegö, Orthogonal polynomials, 3rd ed., Amer。 Math. Soc. Colloq. Publ., vol. 23, Amer. Math. Soc., Providence, R. I., 1967.

13. G. N. Watson, A treatise on the theory of Bessel functions, 2nd ed., Cambridge Univ. Press, New York, 1966.

14. A. Zygmund, Trigonometric series. Vols. I, II, 2nd ed., Cambridge Univ. Press, New York, 1968. MR $38 \# 4882$.

DEPARTMENT OF MATHEMATICS, SAINT PETER'S COLLEGE, JERSEY CITY, NEW JERSEY 07306 (Current address)

DEPARTMENT OF MATHEMATICS, RUTGERS UNIVERSITY, NEW BRUNSWICK, NEW JERSEY 08903 